\title{
"Die Rache der Antikommunisten war nicht minder grausam." Die Ereignisse von 1965/66 in der indonesischen Erinnerungs- kultur und ihre Rezeption im deutschsprachigen Raum
}

\author{
Andreas Fink \\ Kerngebiet: Zeitgeschichte \\ eingereicht bei: assoz. Prof. Mag. Dr. Eva Pfanzelter (MA) \\ eingereicht im: SS 2017 \\ Rubrik: SE-Arbeit
}

\begin{abstract}
“The anticommunists' revenge was no less cruel." The events of 1965/66 within the Indonesian culture of remembrance and its German media coverage

This seminar paper aims to demonstrate the localization of "1965/66" within the Indonesian culture of commemoration and its function for Suharto's politics of history; it shows the narrative's continuities and changes and highlights the German media reception. Therefore it aims to reconstruct the regime's official "PKI coup" narrative with the diorama descriptions at the Pengkhinatan PKI museum (Jakarta) and puts it in contrast to the media coverage in the news magazine Der Spiegel to discuss the recent changes in the transnational culture of remembrance.
\end{abstract}

\section{Einleitung: Indonesiens erfundene Geschichte}

Eine Nation, so der vor zwei Jahren verstorbene Politikwissenschaftler und IndonesienExperte Benedict Anderson (1936-2015), sei eine imaginierte Gemeinschaft; es sei niemals möglich, alle Mitglieder zu kennen und dennoch existiere "in the minds of each [...] the image of their communion"1. Dieser Ausdruck evoziert jenen der Erscheinung, muss aber im Sinne einer schöpferischen Vorstellung verstanden werden. Homi K.

Benedict Anderson, Imagined Communities. Reflections on the Origin and Spread of Nationalism, London-New York 2006, S. 6. 
Bhaba ergänzt: Sie ist eine erzäh/te Gemeinschaft, die durch geteilte Narrative konstituiert wird. ${ }^{2}$ Vorgestellt und erzählt geht das Stiften von Gemeinschaft stets mit der Produktion von Ausschlüssen einher.

Auch für die indonesische Gesellschaft ist dies zutreffend: Die Vorstellungen und Erzählungen, die dieser Gemeinschaft zu Grunde lagen, schlossen die Erfahrungen und Eindrücke von Millionen von Indonesier_innen aus, hatten sie jahrzehntelang unaussprechbar gemacht. Es sind traumatische Erfahrungen: Mord, Folter, Vertreibung, Vergewaltigung, Ausgrenzung. Erfahrungen, die nur langsam zum Teil des kollektiven Gedächtnisses der Gesellschaft werden.

Drei Jahrzehnte regierte der ehemalige General Haji Mohamed Suharto (1921-2008, Staatspräsident von 1967 bis 1998) diktatorisch das Land, an die Macht gekommen im Zuge der Niederschlagung des vermeintlichen Umsturzversuches der sich Gerakan September Tiga Puluh (Bewegung 30. September, G30S) nennenden Gruppe von Offizieren am 1. Oktober 1965. ${ }^{3}$ Die daran anschließenden Ereignisse folgten der Logik einer Schock-Strategie und hatten aufgrund ihrer skalaren Ausweitung massive Auswirkungen auf die gesamte Gesellschaft: Die für den vermeintlichen Putsch verantwortlich gemachte Kommunistische Partei Indonesiens (PKI) wurde zerschlagen, den Anführern der Prozess gemacht, Parteimitglieder und Sympathisant_innen in einer das ganze Land erfassenden Säuberungswelle massakriert und vertrieben.

Machtergreifung und -erhalt des Suharto-Regimes hatten von Beginn an zwei Seiten: Zum einen behauptete Suharto seine Vorherrschaft durch Eliminierung der politischen Gegner_innen, durch ihre Verhaftung und ihren Ausschluss aus dem politischen und sozialen Leben. Zum anderen vollzog sich diese durch die Schaffung eines legitimationstechnisch wirkmächtigen propagandistischen Narrativs und infolgedessen einer spezifischen Erinnerungspolitik und -kultur. In diesem Framing wurden die Ereignisse von 1965/66 zur "Rettung des Vaterlandes" vor dem „kommunistischen Komplott" verklärt und als Schlüsselmoment der historischen Entwicklung und Gründungsmythos für das national-autoritäre, marktliberale Gesellschaftsprojekt der Orde Baru („Neue Ordnung") konstituiert. Auch zwanzig Jahre nach dem Rücktritt Suhartos am 21. Mai 1998 ist dieses Narrativ in Indonesien wirkmächtig und dominiert die offizielle Geschichtserzählung. Gleichzeitig konnten sich seitdem alternative Sichtweisen auf die Ereignisse von 1965/66 erfolgreich etablieren, ihre Anerkennung stellt für die indonesische Gesellschaft jedoch noch immer eine große Herausforderung dar.

In dieser Arbeit soll versucht werden, die spezifische Einschreibung der Chiffre "1965/66" in die Erinnerungskultur und -politik des Landes und den Wandel derselben zu illustrieren. Zunächst wird das offizielle „PKI-Putsch“-Narrativ rekonstruiert und mit dem aktuellen Forschungsstand abgeglichen, ehe aufgezeigt werden kann, inwieweit die Rezeption der Ereignisse im deutschsprachigen Raum dieser Erzählweise folgt.

2 Homi K. Bhabha (Hrsg.), Nation and Narration, London-New York 1990, S. 1-2.

3 John Roosa, Pretext for Mass Murder. The September 30th Movement and Suharto's Coup d'État in Indonesia, Madison 2006. 
Dazu wird zunächst eine Skizze des theoretischen Zugangs geboten und insbesondere das Verhältnis von Geschichte, Diskurs und Gedächtnis nachgezeichnet. Im Anschluss erfolgt eine Kontextualisierung der Fragestellung und die Rekonstruktion der offiziellen Darstellung des "PKI-Putschs" anhand einer Diskursanalyse der Dioramen-Texte des Museums Pengkhianatan PKI (Teil des Pankasilia-Sakti-Museumskomplexes in Jakarta), die im Gegensatz zum Relief und dem Film „Pengkhianatan G30S/PKI" (1984) von Arfin C. Noer noch wenig Aufmerksamkeit erfahren haben. ${ }^{4}$ Dies soll durch einen Vergleich mit der Darstellungsweise in deutschsprachigen Medien Rückschlüsse auf mögliche Ursachen von Kontinuität oder Verschiebung im Diskurs ermöglichen. Hierzu wird - exemplarisch - die Berichterstattung im Nachrichtenmagazin Der Spiegel am Beginn (Untersuchungszeitraum 1965-67) und Ende des Regimes bzw. in der PostSuharto-Zeit (1990-2017) einer kritischen Analyse unterzogen.

Forschungsleitend sei zum einen die These, wonach das Narrativ des „PKI-Putschs" bereits eine bedeutende Rolle bei der Machtergreifung Suhartos spielte und infolgedessen sukzessive und gewaltförmig in der diskursiven Erinnerungspraxis verankert wurde. Erst ab 1998 konnten Gegennarrative öffentlich artikuliert und institutionalisiert werden, wobei das Putsch-Narrativ auch nach zwanzig Jahren noch immer im sozialen und politischen Leben Indonesiens greifbar ist. Zum anderen geht diese Arbeit davon aus, dass auch die Rezeption der Ereignisse durch deutschsprachige bürgerlich-liberale Medien in den 60er-Jahren unter der Deutungshoheit des Putsch-Narrativs stand. Erst mit dem Ende des Kalten Krieges und der Sichtbarmachung der Missstände im Land im Zuge der zum Sturz Suhartos führenden Proteste in den 1990er-Jahren setzte eine Diskursverschiebung ein, die sich durch das Medienereignis, das der Dokumentarfilm "The Act of Killing" (2012) ${ }^{5}$ von Joshua Oppenheimer darstellt, verstetigte.

Der Thematik wird in der angelsächsischen Scientific Community im Gegensatz zur deutschsprachigen breite Aufmerksamkeit zuteil, insbesondere in den letzten zwei Jahrzehnten ist eine Fülle an Publikationen dazu erschienen. Hervorzuheben sind die Arbeiten von Harold Crouch ${ }^{6}$ und John Roosa ${ }^{7}$ zu Ablauf und Hintergründen des „Putschversuchs" und jene von Katharine E. McGregor ${ }^{8}$ und Ariel Heryanto ${ }^{9}$ zur Konstruktion der Erinnerungspolitik unter Suharto und ihren Folgen für die Entwicklung des Landes. Die einzig nennenswerte jüngere deutschsprachige Publikation, das von Anett Keller 2015 herausgegebene Werk "Indonesien 1965ff."10, beleuchtet die Nachwirkungen der Ereignisse von 1965/66 in der indonesischen Gegenwartsgesellschaft. Erwähnenswert ist zudem die 2017 eingereichte, noch unveröffentlichte Masterarbeit

4 Siehe hierzu die Analysen von Relief und Film in: Katharine E. McGregor, History in Uniform. Military Ideology and the Construction of Indonesia's Past, Singapore 2007, S. 75-84 bzw. S. 96-100.

5 Joshua Oppenheimer/Signe Byrge Sørensen, The Act of Killing, DVD, 159 min. Dänemark-NorwegenGroßbritannien 2012.

Harold Crouch, The Army and Politics in Indonesia, Singapore 2007.

Roosa, Pretext for Mass Murder.

McGregor, History in Uniform.

Ariel Heryanto, State Terrorism and Political Identity in Indonesia. Fatally Belonging, London-New York 2006.

10 Anett Keller (Hrsg.), Indonesien 1965ff. Die Gegenwart eines Massenmordes. Ein politisches Lesebuch, Berlin 2015. 
von Paula Hendrikx, die das Fortwirken der unter Suharto institutionalisierten Erinnerungskultur analysiert. ${ }^{11}$

\section{Vergangenheit, Diskurs und Erinnerung}

Mit Halbwachs kann zunächst von einer grundlegenden Unterscheidung zwischen dem Faktualen der Vergangenheit (die Summe vergangener Ereignisse, die durch Quellen und die Wahrnehmung von Zeitzeug_innen rekonstruierbar ist) und dem gegenwärtigen Erinnern ausgegangen werden. ${ }^{12}$ Zwischen dem Vergangenen und dem Sprechen darüber klafft eine unüberbrückbare Lücke, sodass jeder Versuch der Annäherung zwangsläufig selektiv und rekonstruktiv ist. Dazwischen, so Halbwachs, ist die Geschichte zu situieren, das "Verzeichnis der Geschehnisse, die den größten Raum im Gedächtnis der Menschen eingenommen haben"13. Diese Geschichtsschreibung versuche, „eine Brücke zwischen Vergangenheit und Gegenwart zu schlagen"14, bleibt dabei jedoch stets "Sache nur einer ganz geringen Minderheit"15. Sie ist zudem „ungeteilt"16, d. h. ein großes Ganzes, das sich zwar ausdifferenzieren lässt, aber von Eindeutigkeit geprägt ist. Halbwachs, der hier eine „objektiv[e] und unparteiisch[e]"17 Geschichtswissenschaft vor Augen hat, kann nicht gänzlich zugestimmt werden: Plausibler im Sinne einer selbst-kritischen Historiographie ist es, diese als Teil der gesamtgesellschaftlichen diskursiven Auseinandersetzung mit Vergangenem zu betrachten und damit im Feld des Vieldeutigen, Widersprüchlichen und Konflikthaften zu belassen.

Diskurse sind nach Foucault als diskursive Praxis zu verstehen und umfassen verbalisierte und textuelle Äußerungen ebenso wie räumliche Repräsentationen, soziale Praktiken und Rituale, die das Wissen über die Vergangenheit in unterschiedlicher Art und Weise strukturieren und institutionalisieren. Das - nach Jan und Aleida Assmann in kommunikatives und kulturelles zu unterscheidende ${ }^{18}$ - Gedächtnis ist demzufolge das Produkt dieser diskursiven Praxis, eine temporäre, um Kohärenz bemühte Verdichtung historischen Wissens, die zugleich immer auch als Archiv fungiert, in dem Sinn, als das geteilte Gedächtnis zu Ausgangspunkt und Voraussetzung von Debatte und Handlung wird. Auf diese Art und Weise sind Diskurs und Gedächtnis als Pole zu begreifen, die sich gegenseitig bedingen und ähnlich wie das Begriffspaar Diskurs/ldeologie in einem dialektischen Verhältnis zueinander stehen. Jan Assmann spricht hierbei von „Organisationsform" und sieht in Ritus und Fest die „primäre[n] Organisationsform[en] des kulturellen Gedächtnisses", die durch Partizipation und Wiederholung „die Vermittlung und Weitergabe des identitätsstiftenden Wissens und damit [...] die Reproduktion der

\footnotetext{
11 Paula Hendrikx, „1965“ in Indonesia today. The Politics of Memory in Post-Suharto Indonesia and The Perceptions of the 1965-66 Past among High School Students in Yogyakarta in 2016, Dipl. phil. Leiden 2017.

12 Maurice Halbwachs, Das kollektive Gedächtnis, Frankfurt a. M. 1991, S. 63-64.

13 Halbwachs, Das kollektive Gedächtnis, S. 66.

14 Ebd., S. 67.

15 Ebd.

16 Ebd., S. 72-73.

17 Ebd., S. 72

18 Jan Assmann, Das kulturelle Gedächtnis. Schrift, Erinnerung und politische Identität in frühen Hochkulturen, München 2000, S. 64.
} 
kulturellen Identität" ermöglichen. ${ }^{19}$ Aleida Assmann hat sich in „Erinnerungsräume" ausführlich mit räumlichen Repräsentationen befasst, ${ }^{20}$ während die mit Jürgen Link in Spezial-, Inter- und Elementardiskurse ${ }^{21}$ einzuteilenden sprachlichen und textuellen Äußerungen schon länger Gegenstand der kulturwissenschaftlichen Gedächtnisforschung sind (Tabelle 1).

Im Folgenden soll zunächst der historische Kontext der Ereignisse von 1965/66 rekonstruiert werden, um anschließend anhand dieser konzeptuellen Skizze die Institutionalisierung und den Wandel der indonesischen Erinnerungskultur aufzuzeigen.

Tabelle 1: Übersicht Vergangenheit, Diskurs, Gedächtnis; eigene Darstellung

\begin{tabular}{|c|c|c|c|c|c|}
\hline \multicolumn{2}{|c|}{ Vergangenheit } & $>>$ & Diskurse & $<->$ & Gedächtnis \\
\hline \multirow{3}{*}{ Ereignisse } & Sachquellen & \multirow{3}{*}{ 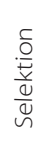 } & Äußerungen & \multirow{3}{*}{ 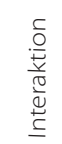 } & kulturell \\
\hline & Textquellen & & Repräsentationen & & kommunikativ \\
\hline & Wahrnehmung & & Rituale & & individuell \\
\hline
\end{tabular}

\section{Die Machtergreifung Suhartos - ein Forschungsüberblick}

Die Ereignisse im Zeitraum vom September 1965 bis zum März 1966 (mit unmittelbaren Nachwirkungen bis März 1967) in Indonesien sind im Kontext des Kalten Krieges und der innenpolitischen Machtkämpfe begreifbar. Präsident Sukarno (1901-1970, Präsident von 1945 bis 1967) verstand es, die Interessensgegensätze der drei großen Lager (muslimische Organisationen, soziale Bewegungen/PKI sowie nationalistisches Lager/Militär) in einem relativ stabilen Machtgleichgewicht auszutarieren und in das Nasakom-Projekt 22 einzubinden. Erst am Ende seiner Regierungszeit verschärften sich die Konflikte wieder und kulminierten in den Ereignissen von 1965/66. Hierbei sind drei verwobene historische Prozesse zu unterscheiden: Zum einen die Aktionen ${ }^{23}$ der Bewe-

19 Assmann, Das kulturelle Gedächtnis, S. 57.

20 Aleida Assmann, Erinnerungsräume. Formen und Wandel des kulturellen Gedächtnisses, München 1999.

21 Anne Waldschmidt/Anne Klein u. a., Diskurs im Alltag - Alltag im Diskurs: Ein Beitrag zu einer empirisch begründeten Methodologie sozialwissenschaftlicher Diskursforschung, in: Forum Qualitative Sozialforschung / Forum: Qualitative Social Research 8 (2007), Heft 2, [http://nbn-resolving.de/urn:nbn:de:0114-fas0702156], eingesehen 3.7.2017.

22 Der Name des Projekts setzt sich zusammen aus den Begriffen nasionalisme, agama, komunisme ("Nationalismus", "Religion”, „Kommunismus"). Suharto, erster Präsident des unabhängigen Indonesien, etablierte Pancasila als Staatsideologie. Diese ist eine heterogene Programmatik, vergleichbar mit den "sechs Pfeilen“ des Kemalismus in der Türkei und dem Peronismus in Argentinien. Gemein ist ihnen die konsensuale Einbindung verschiedener Lager in ein Projekt, das von einer zentralen Persönlichkeit dominiert wird; Pancasila stützt sich auf die fünf Prinzipien von einer Göttlichkeit, Internationalismus, nationaler Einheit, Demokratie und sozialer Gerechtigkeit. Zudem wandelte Suharto das parlamentarische System in ein zentralistisch-autoritär geführtes um - ab 1959 begann die Phase der "gelenkten Demokratie“. Innenpolitisch auf die drei Lager gestützt, näherte er sich später immer mehr dem maoistischen China an und verfolgte einen antiimperialistisch-sozialistischen Kurs: Merle C. Ricklefs, A History of Modern Indonesia since c. 1200, Basingstoke 20013, S. 312-341; Andre Vltchek, Indonesia. Archipelago of Fear, London 2012.

23 Die Bezeichnung dieses Prozesses variiert je nach Einschätzung der Involvierten und ihrer Absichten; die Forschungsdebatte dreht sich um die Frage, ob die Ereignisse als innermilitärischer Konflikt oder eher als Putschversuch (mit dem Ziel, die Regierung zu stürzen) zu deuten sind. „The assasination of the generals [...] was not really a coup attempt against the government, but the event has been almost universally described as an ,abortive coup attempt', so I have continued to use the term.", schreibt Harold Crouch in "The Army and Politics in Indonesia”, (S. 101). Frederick Bunnell setzt die Bezeichnung "coup“ in Anführungszeichen, siehe: 
gung 30. September in der Nacht zum 1. Oktober 1965, bei denen sechs der zwölf Mitglieder des Generalstabs der indonesischen Streitkräfte ausgeschaltet wurden. Zum anderen der Militärputsch des Generals Suharto, der mit umfassenden Repressionsmaßnahmen die Macht übernahm und das politische Projekt der Orde Baru installierte. Darin eingebettet, jedoch mit einer gewissen Eigendynamik ablaufend, sind drittens die Massaker von 1965/66 zu sehen, bei denen je nach Schätzung bis zu einer Million Indonesier_innen getötet wurden. ${ }^{24}$ Für eine ausführliche Rekonstruktion dieser Prozesse sei auf die bereits vorhandene Forschungsliteratur verwiesen. ${ }^{25}$ Im Folgenden soll jedoch die für die weitere Entwicklung relevante Rolle der PKI beleuchtet werden, da diese im hegemonialen Narrativ des Suharto-Regimes als Drahtzieherin des „Putschversuches" dargestellt wird.

Benedict Anderson und Ruth McVey kamen in ihrer als "Cornell Paper"26 bekannt gewordenen Studie von 1966/71 zu einem anderen Schluss: Die Aktionen der Bewegung 30. September "worked against rather than for the interests of the PKI“27. Diese hatte „"no demonstrable cause to plan or join in the September 30th Movement" ${ }^{\prime 28}$. Die Kommunist_innen seien, so Anderson und McVey, organisatorisch nicht involviert gewesen. Vielmehr handelte es sich um einen innermilitärischen Konflikt, bei dem Offiziere aus den mittleren Rängen eigenständig gegen die rechte, US-freundliche Militärführung agiert und auf die spontane Unterstützung von Präsident Sukarno gehofft hätten. ${ }^{29}$ Dieser vorläufigen These widersprachen spätere Äußerungen von Beteiligten teilweise, sodass Crouch sie als "very difficult to defend in its original form"30 bezeichnet und "the involement of the PKI in one way or another" als "widely accepted" betrachtet. ${ }^{31}$ Es kann davon ausgegangen werden, dass die Bewegung um die aufständischen Offiziere heterogen zusammengesetzt war und progressive wie auch konservative Militärs umfasste. ${ }^{32}$ Christian Gerlach betont,

Frederick Bunnell, American „Low Posture" Policy toward Indonesia in the Months Leading up to the 1965 "Coup", in: Indonesia 25 (1990), Heft 50, S. 29-60, hier S. 29. Auch Ariel Heryanto verweist in ,State Terrorism' (S. 7-8) auf die Problematik. Da diese Frage an dieser Stelle nicht annähernd beantwortet werden kann, wird versucht, eine neutrale Terminologie zu verwenden.

24 Neuere Forschungen geben die Opferzahlen mit 0,5 bis eine Mio. Toten an, siehe Leslie Dwyer/Degung Santikarma, „When the World Turned to Chaos". 1965 and its Aftermath in Bali, Indonesia, in: Robert Gellately/ Ben Kiernan (Hrsg.), The Specter of Genocide. Mass Murder in Historical Perspective, Cambridge 2003, S. 289-306, hier S. 290. Dies macht bei einer geschätzten Gesamtbevölkerung Indonesiens im Jahr 1965 von 110 Mio. rund 0,5 bis $1 \%$ aus, siehe hierzu U.S. Bureau of the Census, World Population 1979. Recent Demographic Estimates for the Countries and Regions of the World, Washington D. C. 1980, S. 198. Die Opferzahlen bewegen sich damit in einer ähnlichen prozentualen Größenordnung wie das ebenso antikommunistisch motivierte Bodo-LeagueMassaker in Südkorea von 1950 (0,1 bis 0,2 Mio. Tote bei einer Bevölkerung von 20 Mio.). Die absoluten Zahlen sind vergleichbar mit jenen des Genozids an den Armenier_innen (0,3 bis 1,5 Mio) und dem in Ruanda (0,8 Mio.).

25 Crouch, The Army and Politics, S. 97-134; Roosa, Pretext for Mass Murder.

26 Benedict Anderson/Ruth McVey, A Preliminary Analysis of the October 1, 1965, Coup in Indonesia, Singapore 2009. Diese als „Cornell Paper" bekannt gewordene, im Januar 1966 abgefasste und fünf Jahre später veröffentlichte Studie beschäftigte sich als erste aus einer wissenschaftlichen Perspektive mit den Hintergründen der Ereignisse und widersprach der offiziellen Version in den meisten Punkten.

27 Anderson/McVey, Preliminary Analysis, S. 158.

28 Ebd., S. 163.

29 Ebd.

30 Crouch, The Army and Politics in Indonesia, S. 101.

31 Ebd., S. 104.

32 Andreas Ufen, Vergangenheitspolitik in Indonesien: Die Massaker von 1965-1966, in: GIGA Focus Asien (2014), Heft 3, S. 3. 
"all theories about various string-pullers and allegedly sophisticated coup plotters have remained unsubstantiated, and leave the impression that nobody had a clear plan." ${ }^{\prime 33}$

Selbst jene Wissenschaftler_innen, die einen maßgeblichen Einfluss von Kommunist_innen auf die Geschehnisse konstatieren, können die These eines dezidiert der PKI anzulastenden Putschs nicht bestätigen. Zwar sei, so John Roosa, die Koordinierung oben genannter Gruppen und Einzelpersonen dem damaligen Vorsitzenden der PKI Dipa Nusantara Aidit (1923-1965) und dessen Mitarbeiter Kamaruzaman Sjam (1924-1986) zuzuschreiben: Die Faktenlage „indicate[s] that Aidit collaborated with Sjam to organize the movement as a preemptive strike against the right-wing army high command." ${ }^{34}$ Diese beiden Akteure, Aidit und Sjam, hätten jedoch als Einzelpersonen agiert: „[I]t points to the culpability of only Aidit and Sjam, not the entire party leadership“35, oder - wie hinzufügt werden muss - der Partei als Gesamtorganisation. "[A]nyone but Aidit and a handful of his trusted comrades were in some way complicit [...]"36, bilanziert Roosa. Durch die Inhaftierung des Generalstabs, so die wahrscheinliche Motivation, sollte einem Militärschlag zuvorgekommen und der politische Einfluss vergrößert werden - ein Plan, der aufgrund mangelhafter Organisation und Koordination aus dem Ruder lief und bereits am 2. Oktober 1965 in sich zusammenbrach. ${ }^{37}$ Vor allem aber strebten die beiden keinen Umsturz oder eine Machtübernahme an: Nach Roosa waren die Aktionen "a putsch against the army high command", kein Staatsstreich oder revolutionärer Aufstand. ${ }^{38}$

Die darauffolgenden Ereignisse, die zahlreiche historische und zeitgenössische Parallelen aufweisen (etwa die Machtergreifung von General Augusto Pinochet in Chile 1973 oder der Putschversuch in der Türkei 2016), lassen sich am besten als „SchockStrategie“39 beschreiben: Die Armeeführung „consciously exploited the opportunity provided by the coup attempt to liquidate the PKI leadership."40 Die PKI, vom Militär als alleinige Verantwortliche des „Putschversuchs" denunziert, wurde systematisch vernichtet und am 12. März 1966 verboten, zeitgleich mit der de-facto-Machtübernah-

33 Christian Gerlach, Extremely Violent Societies. Mass Violence in the Twentieth-Century World, Cambridge 2010, S. 19.

34 Roosa, Pretext for Mass Murder, S. 203.

35 Ebd.

36 Ebd., S. 224.

37 Roosa geht davon aus, dass die Generäle lediglich verhaftet werden und vor Sukarno als enttarnte Putschisten präsentiert werden sollten, dieser sollte Aktion und Bewegung danach gutheißen. Nachdem jedoch drei davon bei der Ergreifung durch unerfahrene Kommandos getötet wurden und einer fliehen konnte, exekutierte man auch die anderen. Sukarno, der dies nicht billigen konnte, verweigerte seine Unterstützung, woraufhin die beteiligten Offiziere die Aktion für gescheitert betrachteten. Aidit und Sjam versuchten noch, die Bewegung alleine weiterzuführen und hierzu Partei und Bevölkerung zu mobilisieren, dies misslang ebenso. Als Suharto zum Gegenschlag ausholte, war die Bewegung bereits im Zerfall begriffen; siehe Roosa, Pretext for Mass Murder, S. 217-220.

38 Ebd., S. 224.

39 Der Begriff geht auf Naomi Klein zurück, die in ihrem gleichnamigen populärwissenschaftlichen Werk die Ausnutzung von Krisen- und Katastrophensituationen zur Durchsetzung neoliberaler Agenden in verschiedenen Ländern beschreibt. Zu Indonesien siehe: Naomi Klein, The Shock Doctrine. The Rise of Disaster Capitalism, New York 2007, S. 123-127.

40 Crouch, The Army and Politics, S. 135. 
me durch Suharto, die ein Jahr später auch formal vollzogen wurde. Das Orde Baru genannte autoritäre Gesellschaftsprojekt, das ab 1967 umgesetzt wurde und bis zum Rücktritt Suhartos 1998 Bestand hatte, kann als nationalistische, die Pancasila-ldeologie forcierende Militärdiktatur beschrieben werden, die "extreme market 'reforms'"41 und eine enge Bindung an die USA und das "westliche Lager" verfolgte. Blutiger Gründungsakt dieses Projekts war die systematische Ermordung und Verfolgung von hunderttausenden Indonesier_innen, die als „Feinde der Nation“42 gebrandmarkt wurden. Das Spektrum der Repressionsmaßnahmen ${ }^{43}$ reichte von Tötungen auf offener Straße und systematischen Exekutionen über Verhaftungen und Internierungen in Arbeitslager bis hin zur sozialen Ausgrenzung und Stigmatisierung vermeintlich Involvierter. ${ }^{44}$ Es steht außer Frage, dass sich durch die Aktionen der Bewegung 30. September der politische Konflikt ins Feld des Militärischen verschob und dort von den rechtsgerichteten Kräften um Suharto gewaltsam entschieden wurde.

Für die Durchsetzung und Behauptung eines Gesellschaftsprojektes bedarf es jedoch, folgen wir Gramsci, ${ }^{45}$ mehr als nur roher Zwangsmaßnahmen: Breite Bevölkerungsschichten müssen durch konsensuale Mittel wie Zugeständnisse und Deutungsangebote in das Staatsprojekt eingebunden werden. Medien, Erziehungs- und anderen Institutionen der Zivilgesellschaft obliegt die zentrale Rolle der Produktion von Konsens und der Erlangung der Deutungshoheit durch die Schaffung eines kohärenten, weithin geteilten Sinnhorizontes. Suharto setzte von Beginn an auf die Beeinflussung der öffentlichen Meinung und der Medien ${ }^{46}$ und vermochte sich dadurch nicht nur gegenüber seinen politischen Kontrahent_innen durchzusetzen, sondern auch den Fortbestand seiner Herrschaft über Jahrzehnte abzusichern. Zentrales Element dabei war die Hegemonialisierung des Narrativs zu den Ereignissen von 1965/66.

\section{4. „Putsch der PKI“ - das Narrativ und seine Funktion}

Das auf Hayden White, Claude Lévi-Strauss und Jean-Francois Lyotard zurückgehende Konzept des grand, meta oder master narrative (deutsch "Meistererzählung" oder "Metanarrativ") 47 erscheint hilfreich in der Benennung der sich ab 1965 herausbildenden Diskurskonstellation und bezeichnet im geschichtswissenschaftlichen Kontext

41 Vltchek, Indonesia, S. 44

42 Vorrangig Funktionär_innen, Mitglieder und Sympathisant_innen der PKI, aber auch Sukarno-Anhänger_innen, Intellektuelle, Linke und Angehörige ethnischer Minderheiten wurden zur Zielscheibe der Verfolgungen und Tötungsaktionen.

43 Aufgrund divergierender Machtverhältnisse gab es keinen direkten "Vernichtungsbefehl“; die Anordnung zur Zerschlagung der PKI wurde informell in die Provinzen hinausgetragen, weshalb es in der Umsetzung große regionale Unterschiede gab, siehe: Crouch, The Army and Politics, S. 142. Nichtsdestotrotz sind in den divergierenden Vorgangsweisen Muster erkennbar, die auf eine Systematik verweisen: Hauptakteure waren reguläre Truppen und paramilitärische Einheiten, die in Kollaboration mit nationalistischen und muslimischen (Partei)Organisationen (zum Teil Jugend- und Studierendenverbände) und Angehörigen der Zivilgesellschaft die Massaker verübten.

44 Einen guten Einblick in die Dimensionen der Repression liefert Keller, Indonesien 1965ff.

45 Harald Neubert, Antonio Gramsci: Hegemonie - Zivilgesellschaft - Partei. Eine Einführung, Hamburg 2001; Florian Becker, Gramsci lesen. Einstieg in die Gefängnishefte, Hamburg 2014.

46 Gerlach, Extremely Violent Societies, S. 23.

47 Hayden White, Metahistory. Die historische Einbildungskraft im 19. Jahrhundert, Frankfurt a. M. 1991; Jean Francois Lyotard, Das postmoderne Wissen. Ein Bericht, Graz 1986. 
„eine kohärente, mit einer eindeutigen Perspektive ausgestattete und in der Regel auf den Nationalstaat ausgerichtete Geschichtsdarstellung, deren Prägekraft nicht nur innerfachlich schulbildend wirkt, sondern öffentliche Dominanz erlangt." ${ }^{\prime 4}$

Mit einer ähnlichen auf James Cliffords Skript-Begriff zurückgehenden Definition arbeitet Heryanto, der darunter "a canon, on the basis of which a potentially endless exegetical discourse can be generated"49, versteht. Dieses dominante Narrativ ist Produkt einer diskursiven Auseinandersetzung und wird als verdichtete, hegemonial gewordene Kernbotschaft in unterschiedlichen Diskursen reproduziert, weshalb die Analyse klären muss, welches Aussagesetting es umfasst, wie es in der alltäglichen diskursiven Praxis manifest wird, welche Funktion es erfüllt und wie es sich gegen konkurrierende Deutungsoptionen durchsetzen konnte und kann. Dies soll im Folgenden versucht werden.

Die Genese des später zum master narrative gewordenen Diskurses der "verhinderten PKI-Revolution“ lässt sich bis zum 4. Oktober 1965 zurückverfolgen. In der kurzen Rede Suhartos nach der Exhumierung der Leichen der getöteten Generäle stellte dieser als Erster eine direkte Verbindung zwischen den Morden und der PKI her, ${ }^{50}$ "[a]lthough the evidence available in the first few days seemed to indicate that dissident army officers [...] were mainly responsible..51 Damit ging er auf direkten Konfrontationskurs zu Sukarno, der „individuals from all sections“52 für die Ereignisse verantwortlich sah. Durch die Kontrolle der TV-, Radio- und Printmedien war es der Militärführung um Suharto jedoch möglich, nicht nur die diskursive Intervention der Bewegung 30. September („Rettung der Regierung vor einem Putsch der rechtsgerichteten Militärführung“) auszumanövrieren, sondern auch Sukarnos Versuche, die Ereignisse als wenig gewichtigen innermilitärischen Konflikt zu deuten, in den Einzelpersonen aus unterschiedlichen Lagern involviert gewesen seien. Die Exekution der Generäle wurde durch die Militärs um Suharto zum Folterspektakel ${ }^{53}$ deklariert, Auftakt eines gewaltsamen und blutigen Umsturzversuches der PKI und ihrer Vorfeldorganisationen.

Dieses Narrativ fügt in seiner Rekonstruktion der Ereignisse neue Elemente - Handlungen ebenso wie Personen (Folter und Misshandlung der Generäle, Verstümmelung ihrer Leichen, kollektive Zelebrierung der Ermordung, Beteiligung von Parteimitgliedern) - ein. Zudem wird eine doppelte diskursive Verkettung produziert: 1. die Verbindung

48 Konrad H. Jarausch/Martin Sabrow, "Meistererzählung”. Zur Karriere eines Begriffs, in: Konrad H. Jarausch/Martin Sabrow (Hrsg.), Die historische Meistererzählung. Deutungslinien der deutschen Nationalgeschichte nach 1945, Göttingen 2002, S. 16.

49 Heryanto, State Terrorism, S. 8.

50 Crouch, The Army and Politics, S. 138

51 Ebd., S. 139

52 Ebd.

53 Roosa konstatiert: "The Suharto regime's story - that the seven officers were tortured and mutilated by crowds of ecstatic PKI supporters, while women from Gerwani (the Indonesian Women's Movement) danced naked — was an absurd fabrication by psychological warfare experts." (Roosa, Pretext for Mass Murder, S. 40) Und Gerlach: „[C] ivilians from PKI-affiliated organizations in general were not involved in the killing of the generals nor in military action in the coup in Jakarta except for a late attempt to occupy buildings near Merdeka Square." (Gerlach, Extremely Violent Societies, S. 300). 
der Ermordung der Generäle mit Putsch und Revolution, d. h. eine beabsichtigte umfassende Veränderung der politischen und sozialen Verhältnisse; und 2. die Gleichsetzung der Bewegung 30. September mit der PKI als Organisation (unmissverständlich durch die durchgängige Bezeichnung "G30S/PKI“ bzw. "Gestapu“54) und diese mit den Parteimitgliedern und Linken allgemein. Dadurch erzeugt das Narrativ ein Freund-FeindSchema, in dem Suharto und das Militär die Rolle des "Retters" und die PKI die des "Feindes der Nation" einnehmen: The New Order version of history was a simple story of a heroic military and a cruel communist party. ${ }^{155}$ Das Folter-Narrativ wird als Beleg für das Bevorstehende ins Feld gerückt und dient damit unmittelbar der Legitimierung der militärisch-politischen Maßnahmen zur Zerschlagung der PKI als politischer Kraft und ihrer faktischen physischen Vernichtung. Es fungiert „as a means by which the regime defined its core values, including defence of the Pancasila, and a restoration of moral order to society ${ }^{\prime 56}$ - ungeachtet dessen, dass auch am Höhepunkt der Massaker der Öffentlichkeit keine Belege vorlagen, dass die PKI die Bewegung orchestriert hätte. ${ }^{57}$

Die Institutionalisierung des Narrativs erfolgte - wie im Folgenden gezeigt wird - in allen drei Dimensionen des Diskurses und damit der kollektiven Erinnerung der indonesischen Gesellschaft: durch Äußerungen und Textproduktion (wissenschaftliche und populäre Medien), durch Repräsentationen im Raum, den Erinnerungsorten (Museen, Denkmäler, Namenspolitik) sowie mittels Ritualen und Veranstaltungen (Feiertage, Filmvorführungen).

Als Vorsitzender des Zentrums für Geschichte der indonesischen Streitkräfte (1965-85) und Bildungsminister (1983-85) war Nugroho Notosusanto (1930-1985) „,the military's main historian" 58 und "one of the most important propagandists of the New Order regime ${ }^{\prime 59}$, maßgeblich bei der Ausarbeitung und textuellen Verbreitung des master narrative beteiligt. Bereits 1966 publizierte das Zentrum für Geschichte mit „Die 40 Tage des Scheiterns der Bewegung 30. September"60 eine erste, weit verbreitete Rekonstruktion der Ereignisse, in der die PKI als Drahtzieherin genannt ${ }^{61}$ und die Rolle des Militärs bei den Gewaltausbrüchen negiert wurden. ${ }^{62}$ Diese Version wurde als Reaktion auf das "Cornell-Paper" von Anderson/McVey und an ein internationales akademisches Publikum adressiert und in Nugrohos zwei Jahre später publiziertem Werk „The Coup

54 Die der Bewegung von der Presse zugeschriebene propagandistische Bezeichnung "Gestapu“ wurde in Ergänzung zur Chiffre "G30S/PKI" gebraucht und ist ein loses Akronym von Gerakan September Tiga Puluh („Dreißigster September Bewegung"), wobei die in Indonesien übliche Schreibweise umgestellt wurde, um Assoziationen mit der Gestapo (Geheime Staatspolizei) des NS-Regimes zu wecken.

55 Grace Leksana, Reconciliation through history education: reconstructing the social memory of the 1965-66 violence in Indonesia, in: Birgit Bräuchler (Hrsg.), Reconciling Indonesia. Grassroots agency for peace, LondonNew York 2009, S. 175-191, hier S. 178.

56 McGregor, History in Uniform, S. 62.

57 Roosa, Pretext for Mass Murder, S. 64

58 Leksana, Reconciliation, S. 178.

59 McGregor, History in Uniform, S. 39

60 Lembaga Sejarah, 40 hari kegagalan,G-30-S’ 1 Oktober-10 November 1965, Jakarta 1966.

61 McGregor, History in Uniform, S. 63-64.

62 Jess Melvin, Documenting Genocide, in: Inside Indonesia 32 (2015), Heft 122, [http://www.insideindonesia.org/ documenting-genocide-3], eingesehen 10.8.2017. 
Attempt of the September 30 Movement in Indonesia"63 ausgearbeitet, das fortan als Standardwerk der offiziellen Erzählung galt. Es war Grundlage für den von Afrin C. Noer produzierten und 1983 veröffentlichten Kinofilm „Der Verrat der G30S/PKI“64, dem meistgesehenen Film in Indonesien und „perhaps the best-known official representation of the 1965 coup attempt." ${ }^{\prime 65}$ Nugroho war zudem einer der Herausgeber des sechsbändigen „Lehrbuchs zur Geschichte Indonesiens“66 von 1975, der ersten umfassenden Darstellung der Landesgeschichte und „primary reference for history textbooks at school from junior to senior high school." ${ }^{\prime 67}$

Die wichtigste räumliche Manifestation des Putsch-Narrativs stellt das Pancasila-SaktiDenkmal (übersetzt "Heiliges Pancasila") ${ }^{68}$ am Fundort der Leichen der getöteten Generäle im Süden Jakartas in Luban Buaya dar. Die Anlage wurde ab 1967 errichtet, zwei Jahre später eröffnet und später durch zwei Museumskomplexe ergänzt: Das 1981 eingeweihte Pankasilia-Sakti-Museum stellt in neun Dioramen die Ereignisse von 1965 dar, während das von Nugroho initiierte und 1990 eröffnete Museum Pengkhinatan PKI („Museum des PKI-Verrats“) 34 Dioramen zur PKI-Geschichte von 1945 bis 1974 zeigt. $^{69}$

Höhepunkt der ritualisierten kollektiven Erinnerung ist der 1967 eingeführte nationale Gedenktag Hari Peringatan Kesaktian Pancasila (1. Oktober, „Tag der Erinnerung an das heilige/wundersame Pancasila"), an dem der museale Erinnerungsort zum Schauplatz des Gedenkzeremoniells der Staatsspitze und Teil einer umfassenden diskursiven Praxis wird. Zu dieser gehört die Ausstrahlung von Noers Film im Staatsfernsehen am Vortag sowie das "mandatory annual viewing for schoolchildren. ${ }^{170}$

\section{Die Rekonstruktion des master narrative (Dioramen-Text-Analyse)}

Aus diesem Grund sollen im Folgenden die zentralen Elemente des master narrative, wie es im Museumskomplex von Luban Buaya und konkret in den Erklärungstexten der figurativen Dioramendarstellungen manifestiert ist, nachgezeichnet werden. ${ }^{11}$ Die drei Bauphasen der Gedenkstätte - 1969, 1981 und 1990 - spiegeln die Veränderungen im kommunikativen und kulturellen Gedächtnis und den Bedarf einer Kontextualisierung des unmittelbaren Ereignisses (die Gedenkstätte mit Erdloch, Säulen der Generäle) durch die Einbettung in die Erzählung vom PKI-Putsch (das

63 Nugroho Notosusanto/Ismail Saleh, The Coup Attempt of the September 30 Movement in Indonesia, Jakarta 1968.

64 Afrin C. Noer/Nugroho Notosusanto/Gufran Dwipayana, Pengkhianatan G30S/PKI, DVD, 271 min., Indonesien 1984.

65 McGregor, History in Uniform, S. 96

66 Nugroho Notosusanto/Marwati Djoned Poesponegoro u. a. (Hrsg.), Sejarah Nasional Indonesia, Jakarta 1975.

67 Anzar Abdullah, Contemporary History of Indonesia between Historical Truth and Group Purpose, in: Review of European Studies 7 (2015), Heft 12, S. 179-185, hier S. 182.

68 Die zwei wesentlichen Elemente sind eine überdachte Rekonstruktion des Erdlochs, in dem die Leichen gefunden wurden, sowie eines Statuenensambles mit lebensgroßen Bronzestatuen der sieben Generäle unter einem überdimensionalen Geruda-Adler.

69 Siehe Roosa, Pretext for Mass Murder, S. 7-11.

70 Ebd., S. 10

71 Auf eine Einbeziehung der Dioramendarstellung wird verzichtet, diese sind ohne die Erklärtexte vielfach nicht lesbar und unterliegen den Beschränkungen des Mediums (vieles lässt sich mit diesen Mitteln nicht darstellen). 
Sakti-Museum) sowie in die Geschichte der PKI seit 1945 (das Museum Pengkhinatan PKI) wider, wobei das im Säulenrelief dargestellte Kernnarrativ eine Ausschmückung erfährt. Die durch die architektonische Gestaltung intendierte Dekonstruktionspraxis beim Museumsbesuch beginnt bei der Besichtigung von Erdloch, Denkmal und Replika der Folterhütten (inklusive lebensgroßer Nachstellung mit Puppen), führt an den ausgestellten Fahrzeugen auf dem Freigelände vorbei ins Museum Pengkhinatan PKI und endet in dem im selben Gebäude untergebrachten Museumsteil der PankasiliaSakti-Ausstellung, wodurch der Zoom-Out-Effekt (Ereignis > Putsch > PKI-Geschichte) - womöglich bautechnisch bedingt - durchbrochen wird.

Die diskursive Funktion des Museums Pengkhinatan PKI ist die Verbindung des Putschversuchs des Jahres 1965 mit den Maidun-Ereignissen von $1948^{72}$ bzw. der PKI und des Kommunismus mit dem Gewaltmotiv. Wie in untenstehender Abbildung 1 ersichtlich, behandeln 15 von 34 Dioramen die Phase von 1945 bis 1949, wobei am häufigsten das Jahr 1948 (zehn Dioramen) vertreten ist, gefolgt von 1965 (acht Dioramen); vorrangig geht es dabei um Aufstände/Unruhen sowie Tötungen (sieben bzw. vier Dioramen). Den drei Fünf-Jahres-Phasen von 1950 bis 1964 sind drei, null und zwei Dioramen zuzuordnen, während die Phase von 1965 bis 1969 mit elf Dioramen und jene ab 1970 mit zwei vertreten ist.73 Die fünf Darstellungen der Jahre 1950 bis 1965 (diesem sind zwei Dioramen gewidmet) thematisieren kleinere Unruhen, Protestaktionen (ziviler Ungehorsam gegen die Räumung von Ländereien und eine Feldbesetzung), Propagandakampagnen und die Strategie der PKI. Dabei wird der veränderte Charakter der neuaufgestellten Partei ab den 1950ern unterschlagen: Obschon die Maidun-Ereignisse einen fundamentalen Wandel in Führung, Zusammensetzung und Strategie mit sich brachten und die PKI infolgedessen auf einen kooperativen Kurs einschwenkte, ${ }^{74}$ wird versucht, oben genannte Aktionen hinsichtlich ihrer Gewaltförmigkeit zu definieren und eine Kontinuitätslinie zwischen 1948 und 1965 herzustellen. „The first revolt [...]”, so der Museumskatalog, „was launched on 18th September 1948 in Maidun. Having

72 Während des Indonesischen Unabhängigkeitskrieges (1945-1949) kam es in Ost-Java zu einem militärischen Konflikt zwischen rechts- und linksgerichteten Kräften: Die angeordnete Entwaffnung linker Guerilla-Einheiten, die im Unabhängigkeitskrieg mitkämpften, führte 1948 zu einer regionalen Revolte, bei der die Guerilla zeitweilig Gebiete um die Stadt Maidun unter ihre Kontrolle bringen konnte. Der Aufstand wurde von Regierungstruppen nach einigen Wochen blutig niedergeschlagen, führende Köpfe der PKI und tausende Kämpfer_innen getötet oder eingesperrt. Auch bei dieser Episode ist die Akteurskonstellation diffus, die Interpretationsvarianz breit; wesentlich ist sie für die Entwicklung des Antikommunismus in Indonesien und die Verhärtung der Konfliktlinie PKI - Militär. Siehe hierzu The Editors of Encyclopaedia Britannica, Maidun Affair, Encyclopaedia Britannica, 20.1.1998, [https://www.britannica.com/event/Madiun-Affair], eingesehen 20.3.2017; Harry A. Poeze, The Cold War in Indonesia, 1948, in: Journal of Southeast Asian Studies 40 (2009), Heft 3, S. 497-517; Ann Swift, The Road to Maidun. The Indonesian Communist Uprising of 1948, Singapur 2010; McGregor, History in Uniform, S. 52-54; Ricklefs, History of Modern Indonesia, S. 280-282.

73 Siehe Abb. 1 unten.

74 Christie hält fest: „[A]fter the the failed Maidun Revolt of 1948 [...], PKI's very survival depended on a policy based on caution, and accommodation with other elements in the nationalist movement." (Christie, Ideology and Revolution, S. 164) Unter Aidit, der die Parteiführung ab 1951 übernahm, schwenkte die PKI auf einen Kurs ein, der die nationale Befreiung priorisierte und Bildungsarbeit, Massenmobilisierung und Bündnisse mit bürgerlichen Kräften vorsah, siehe Christie, Ideology and Revolution, S. 164-166; Ulla Fionna, The Institutionalisation of Political Parties in Post-authoritarian Indonesia. From the Grass-roots Up, Amsterdam 2013, S. 39-43. 
failed the first revolt, the PKI staged a treachery for the second times [sic] on 30th September 1965 [...]."75

Die sieben letzten Dioramen aus der Zeit nach dem Oktober 1965 stellen Szenen der militärischen und justiziellen Bekämpfung der PKI dar, wodurch sich in der Gesamtdarstellung die schematische Gegenüberstellung von Chaos und Ordnung wiederholt. Ziel ist es, die PKI als grundsätzlich gewalttätig, mörderisch und umstürzlerisch und die Ereignisse von 1965 als logische Konsequenz dieser Charakterisierung darzustellen. Eine Erläuterung der kommunistischen Ideologie und Programmatik unterbleibt gänzlich, der Beitrag der Partei im Unabhängigkeitskampf und ihre nicht-konfrontative Agitation werden unterschlagen. ${ }^{76}$ De facto war die PKI 1965 nicht in der Lage, gegen ihre gewaltsame Zerschlagung und die ausufernden Massaker effektiven militärischen Widerstand zu leisten, da die Parteiorganisation weder auf einen Bürgerkrieg aus noch darauf vorbereitet war. Bemerkenswerterweise werden die Massaker in der musealen Geschichtserzählung völlig ausgeblendet: Die Bekämpfung der PKI und ihrer Vorfeldorganisationen erfolgte, so das Narrativ, durch Verbote, Verhaftungen und Gerichtsprozesse und bewegte sich dabei im Rahmen des Rechts. Selbst bei den drei geschilderten Militäroperationen gegen "Guerillagruppen" ist lediglich von Verhaftungen und einer Erschießung die Rede. ${ }^{77}$

Die zweite Konstruktion, die mit der Verbindungslinie zu 1948 vollzogen wird, ist die Charakterisierung der PKI als „verräterisch“ bzw. anti-national. Seit den Maidun-Ereignissen wurde die $\mathrm{PKI}_{\text {"I }}$ as a traitorous movement that had stabbed the Republic of Indonesia in the back at its moment of greatest peril|"78 denunziert und sah sich mit antikommunistischen Ressentiments konfrontiert. Im Narrativ des Orde-Baru-Regimes wird daran angeknüpft. Die Zentralität dieser Zuschreibung zeigt sich in der entsprechenden Namensgebung des Museumskomplexes (er heißt, analog zu Noers Film, „Museum des Verrats der PKI (Kommunisten)") und wird dort beschrieben als Intrige "against the legal Governmemt of the Republic of Indonesia and [the Pancasila] ideology."79 Neben den Gefahren von außen gelte es, den „inneren Feind“ zu bekämpfen: „One of the threats and obstacles from inside the state was from the Indonesian Communist Party (PKI)."80

75 Markas Besar Tentar Nasional Indonesia Pusat Sejarah/Headquarters of the Indonesian National Military Center for TNI History (Hrsg.), Buku Panduan Monumen Pancasila Sacti/The Guide Book of Pancasila Sakti Monument, Jakarta 2008. In diesem zweisprachigen Katalog bzw. Begleitheft des Museums sind alle Dioramen samt den im Museum angebrachten Erklärtexten abgebildet und durch allgemeine Informationen für Besucher_innen ergänzt.

76 Roosa, Pretext for Mass Murder, S. 10.

77 Bei der Operation Trisula 1968 wurden Tausende ermordet und vertrieben, im Museum ist von elf Verhaftungen die Rede.

78 Clive J. Christie, Ideology and Revolution in Southeast Asia 1900-1980. Political Ideas of the Anti-Colonial Era, Richmond 2001, S. 164.

79 Headquarters of the Indonesian Military Center, Guide Book, S. 5.

80 Ebd., S. III. 


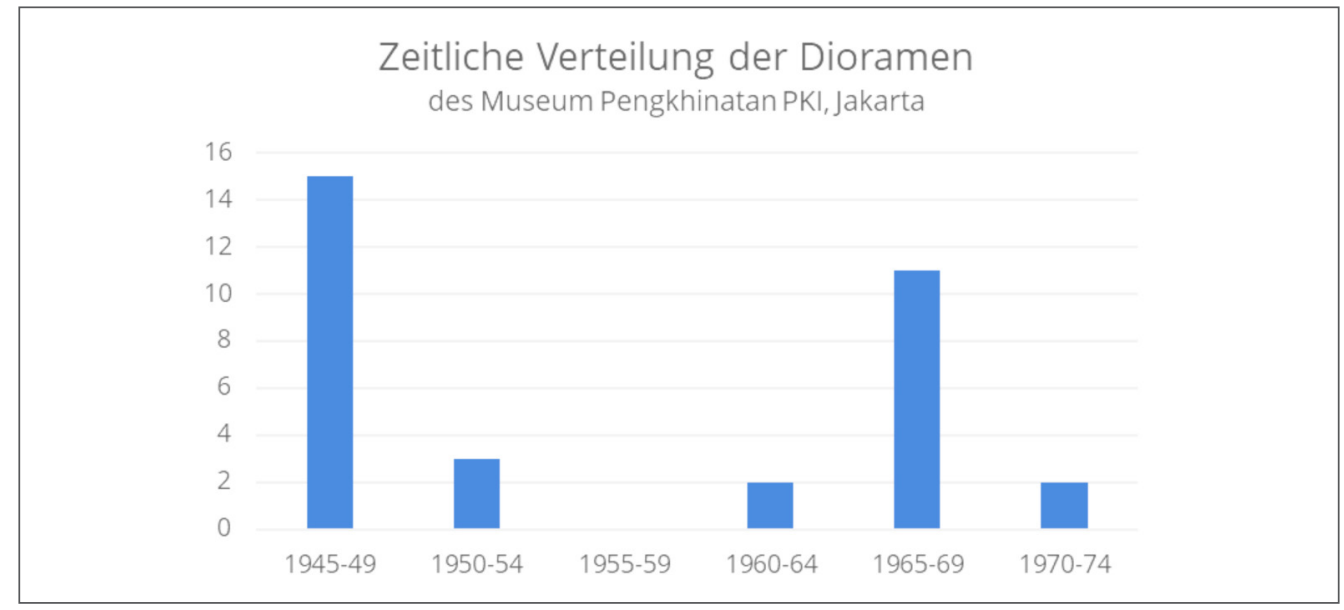

Abbildung 1: Aufteilung der 34 Dioramen des Museums Pengkhinatan PKI (Jakarta) nach Zeitperioden

Auch die Darstellung der Ereignisse vom September und Oktober 1965 in den neun Dioramen des Pankasilia-Sakti-Museums folgt der Logik von Chaos und Ordnung. Wie das erste Diorama deutlich macht, gibt es an der Schuld der PKI keine Zweifel: „On September 1965, the Chairman of Central Committee of the PKI, D.N. Aidit, gave an order to Syam Kamaruzaman, the Chairman or Special Bureau, to make a plan for [a] rebellion." ${ }^{81}$ Den revolutionären Charakter der Aktion und die Beteiligung der gesamten PKI zeigt das zweite Diorama. Bei diesem ist von „many activities to prepare the rebellion" und zwei militärischen Trainings in Luban Buaya die Rede, die von "[c]ommunist young men and women, labours and farmer [sic] as well as other group [sic] in the Communist party" ${ }^{\prime 2}$ abgehalten werden. Der PKI wird unterstellt, sie hätte durch den Putsch die unter Sukarno entstandene Pancasila-Staatsideologie abschaffen und durch die kommunistische ersetzen wollen. Das dritte Diorama zeigt die Erschießung des Armeekommandanten Achmad Yani bei dem Versuch seiner Ergreifung, das vierte die Folterung der gefangenen Generäle in Luban Buya:

"In this house they were maltreated by using some weapons such as guns, knives or other things so that the bodies of the officers were entirely damaged. [...] The maltreatment was done by the members of the PKI's mass organizations such as the People's Youth (PR), the women's movement Gerwani and other PKI organizations." ${ }^{183}$

Im fünften mittleren Diorama tritt Suharto auf. Es stellt die Rückeroberung des Hauptquartiers der putschenden Militärs im Halim-Flughafen dar, während das sechste die Exhumierung der Leichen - „in bad condition because of torture and rot in well" ${ }^{\text {" }}{ }^{\text {- }}$ zeigt. Die Machtübergabe an Suharto 1966 und seine Inauguration als Präsident der Republik 1967, „in order to find a way to solve the problem of the said situation of

81 Headquarters of the Indonesian Military Center, Guide Book, S. 60.

82 Ebd., S. 62.

83 Ebd., S. 64

84 Ebd., S. 67. 
conflict", 85 sind Themen der folgenden beiden Dioramen. Suharto tritt hier als Retter von Präsident und Land auf, der die Ordnung wiederherstellt: „That letter of instruction contained the giving of authority to Lieut. Gen Soeharto to take all steps considered necessary to guarantee security and order, as well as stability in government administration and the course of the revolution." ${ }^{16}$ Abschließend präsentiert die Diorama-Serie eine Wehrschulung ("Tarpnadas“), die 1982 mit dem Ziel eingeführt wurde, die Jugend gegen „the latent danger of communism" ${ }^{\prime 87}$ zu wappnen. Im Stile eines klassischen Dramas mit Exposition, Höhepunkt und Retardation aufgebaut, spannt die Narration so den Bogen bis in die Gegenwart, um das bildungspolitische Ziel der generationenübergreifenden Sinnstiftung zu erfüllen.

Wie sich im Vergleich mit der medialen Berichterstattung im deutschsprachigen Diskurs zeigen wird, gelang die narrative Hegemonialisierung auch im internationalen Kontext: Die Kernelemente des „PKI-Putsch“-Narrativs wurden in der deutschen Presse weitgehend unhinterfragt übernommen.

\subsection{Die mediale Rezeption im deutschsprachigen Raum in den 60ern}

(Der Spiegel-Analyse 1)

Das spärliche Vorhandensein zeitnaher wissenschaftlicher Literatur ${ }^{88}$ legt nahe, dass die Ereignisse von 1965/66 im deutschsprachigen akademischen Diskurs wenig Aufmerksamkeit erhielten. Dies scheint auch für den medialen Diskurs zu gelten, müsste jedoch durch weiterführende Forschungsarbeiten, die die verschiedenen soziopolitischen Milieus berücksichtigen, verifiziert werden. Zur Ermöglichung einer ersten vorläufigen Einschätzung soll im Folgenden die Darstellung im Nachrichtenmagazin Der Spiegel analysiert werden, das heute wie damals vor allem in bildungsbürgerlichen Kreisen rege rezipiert wurde und in der BRD eine stark meinungsbildende Funktion erfüllte. Die Spiegel-Berichterstattung in den knapp eineinhalb Jahren von Oktober 1965 („Putschversuch“) bis Februar 1967 (Rücktritt Sukarnos) umfasst einen Korpus von 15 Artikeln, von denen sich elf vorrangig der indonesischen Innenpolitik widmen. ${ }^{89}$

Die politischen Prozesse und Machtkämpfe standen in den ersten Monaten im Zentrum der Berichterstattung, ab März 1966 verlagerte sie sich und stellte die Rolle Sukarnos und die Verfolgungen in den Mittelpunkt. Auffallend ist, dass die Massaker schon unmittelbar nach ihrem Ausbruch im Oktober Erwähnung fanden: In der Ausgabe vom 27.10.1965 wurden Angriffe auf eine Universität und PKI-Einrichtungen, Lynch-

85 Headquarters of the Indonesian Military Center, Guide Book, S. 70.

86 Ebd., S. 69.

87 Ebd., S. 72

88 Zu erwähnen sind hier - ohne Anspruch auf Vollständigkeit - Flor Peeters, Der Indonesische Kommunismus und der gescheiterte Staatsstreich, in: Zeitschrift für Politik 14 (1967), Heft 2, S. 187-202; Einar Schlereth/Batjo Daeng Bintang, Indonesien. Analyse eines Massakers, Frankfurt a. M. 1970; Philippe Gavi, Konterrevolution in Indonesien, Frankfurt a. M. 1969.

89 Spiegel-Suche, Suchmaskeneingabe "indonesi*“, Zeitraum "alles", "Volltext”, in: Der Spiegel, [http://www.spiegel. de/suche/index.html], abgefragt 3.8.2017. In den anderen fünf Artikeln finden sich Randnotizen dazu: Vier thematisieren die Politik Chinas, im vierten wird Sukarnos Ehefrau Dewi porträtiert. Diese wurden bei der weiteren Auswertung nicht berücksichtigt. Der Korpus-Umfang von 15 Artikeln ist in Relation zu setzen mit rund 75 Ausgaben im besagten Zeitraum (wöchentlich erscheinendes Magazin). 
morde und Erschießungen durch Polizeikräfte geschildert, ebenso Verhaftungen und Hausdurchsuchungen..$^{90}$ Im Januar 1966 berichtete Der Spiege/ von Verhaftungen und Massakern an „nach vorsichtigen Schätzungen [...] Zehntausende[n]"91 in weiten Teilen des Landes. Diese Opferzahl wurde im März mit 100.000 und im Mai mit 87.000 bzw. $500.000^{92}$ angegeben, womit die Untergrenze heutiger Rekonstruktionen getroffen wurde und die Dimension der Massaker abschätzbar war. Im letzten Artikel des Korpus vom Februar 1967 gab das Wochenblatt Schätzungen von 300.000 bis 500.000 Opfern wieder. ${ }^{93}$ Neben dem Agieren Sukarnos befasste sich die Berichterstattung im weiteren Verlauf des Jahres 1966 mit den Prozessen gegen hochrangige Sukarno-Anhänger sowie den Massakern und Verfolgungen, wobei - ähnlich wie in US-Medien ${ }^{94}$ - vor allem die Opferrolle der chinesischen Minderheit betont wurde..$^{95}$ In Formulierungen wie etwa "der Rotenhaß [war] zum Rassenhaß gegen die chinesische Minderheit umgeschlagen" ${ }^{\prime 96}$ sieht Coppel den Versuch der Entpolitisierung, da die massive Gewalt „in racial or primordial terms“ oder durch biologistische Zuschreibungen erklärbar gemacht werde. ${ }^{97}$ Dasselbe lässt sich auch auf der Täterseite ausmachen: Hier wird die Rolle muslimischer Gruppen und Organisationen ${ }^{98}$ sowie von Studierenden und Jugendlichen ${ }^{99}$ - teilweise überlappend bei Begriffen wie "Moslem-Jugend" oder "JungMusulmanen" - bei den Massakern und Übergriffen übermäßig stark betont. Dadurch

90 O. A., Neues Kom, in: Der Spiegel, Nr. 44, 27.10.1965, S. 132-133, [http://www.spiegel.de/spiegel/print/d-46274703. html], eingesehen 11.8.2017.

91 O. A., Macht der Messer, in: Der Spiegel, Nr. 1, 3.1.1966, S. 58, [http://www.spiegel.de/spiegel/print/d-46265207. html] eingesehen 11.8.2017.

92 Nach offiziellen bzw. Diplomaten-Quellen.

93 Dabei handelt es sich um den Abdruck eines Auszuges aus dem Werk "The Fall of Sukarno" des Journalisten Tarzie Vittachi, siehe Tarzie Vittachi, The Fall of Sukarno, New York 1967, [https://archive.org/details/in.ernet. dli.2015.462525], eingesehen 11.8.2017; bzw. Tarzie Vittachi, In Socken floh der Präsident zum Helikopter, in: Der Spiegel, Nr. 10, 27.2.1967, S. 109-120, [http://www.spiegel.de/spiegel/print/d-46394458.html], eingesehen 11.8.2017.

94 Charles A. Choppel, Anti-Chinese Violence in Indonesia after Soeharto, in: Leo Suryadinata (Hrsg.), Ethnic Chinese in Contemporary Indonesia, Singapore 2008, S. 117-136, hier S. 122.

95 Diese wird in vier von sechs Artikeln, die die Massaker und Vertreibungen schildern, erwähnt; im Oktober 1966 ist der gesamte Artikel dieser Thematik gewidmet. Die These vom „Genozid an Chines_innen“ im Zuge der Massaker 1965/66 hält sich bis heute, wird von vielen Forscher_innen jedoch bezweifelt. Charles Choppel „found that the Chinese were underrepresented in the horrific mass killings of 1965-1966"; "the victims of the massacres were overwhelmingly Javanese and Balinese who were identified as communists or communist sympathizers", weshalb die Ereignisse als "Politizid“ zu begreifen seien (Charles A. Choppel, Preface, in: Charles A. Choppel (Hrsg.), Violent Conflicts in Indonesia. Analysis, Representation, Resolution, London-New York 2006, S. XV-XVIII, hier S. XV). Ausführlich wird dies abgehandelt in Robert Cribb/Charles Coppel, A Genocide That Never Was: Explaining the Myth of Anti-Chinese Massacres in Indonesia, 1965-66, in: Journal of Genocide Research 11 (2009), Heft 4, S. 219-239; während für andere Autor_innen der Genozid-Begriff zumindest für die Ereignisse in bestimmten Regionen Indonesiens adäquat erscheint. Jess Melvin findet, „the assessment that the Indonesian killings should not be understood as genocide is premature" (Jess Melvin, Why Not Genocide? Anti-Chinese Violence in Aceh, 1965-1966, in: Journal of Current Southeast Asian Affairs 3 (2013), Heft 32, S. 63-91, hier S. 63).

96 O. A., Frauen töten die Generäle, in: Der Spiegel, Nr. 20, 9.5.1966, S. 99-100, hier 99, [http://www.spiegel.de/spiegel/ print/d-46407207.html], eingesehen 11.8.2017.

97 Coppel, Anti-Chinese Violence, S. 122.

98 Wiederum vier von sechs Artikel über die Massaker thematisieren die Beteiligung muslimischer Parteien und Organisationen, im Buchauszug von Vittachi sind die Massaker ein "Heiliger Krieg” bzw. "Kreuzzug gegen die Ungläubigen".

99 In vier von sechs Artikeln über die Massaker werden Studierende und Jugendliche zu den Hauptakteur_innen gezählt. 
gerät die federführende Rolle der Militärs ${ }^{100}$ aus dem Blick, die Gegenüberstellung irrationaler, massakrierender Muslime/Jugendlicher und rationaler, verhaftender Armee erfüllte eine gleichsam erklärende wie entlastende Funktion.

Beim Vergleich der Darstellungsweise der Vorgänge im Spiegel mit den drei großen Erklärungsnarrativen (G30S, Sukarno, Suharto) zeigt sich eine durchgängige Übernahme der wesentlichen Bestandteile des Suharto-Narrativs. Demzufolge seien die Aktionen der G30S erstens ein Putschversuch der PKI gewesen und hätten zweitens Auftakt eines bewaffneten Umsturzes sein sollen; die Generäle waren drittens brutal gefoltert und misshandelt worden, was viertens zu gewaltsamen Racheaktionen seitens der Bevölkerung geführt habe:

Während Der Spiegel Anfang Oktober noch betonte, es sei „Völlig unklar, wer den Putsch wirklich angestiftet hatte"101, war Ende des Monats bereits von "Mordanschläge[n] roter Putschisten" und einem "von den Kommunisten inspirierten Putsch" die Rede. Im Januar-Artikel wurde schließlich vom "kommunistischen Putsch", der "ein ernst zu nehmender Umsturzversuch" der PKI gewesen sei, berichtet, sogar der diffamierende Kampfbegriff "Gestapu-Putsch“ wird verwendet. ${ }^{102}$ In den Folgeartikeln wird nur mehr von "kommunistischem" oder "rotem Putsch" die Rede sein.

Der "Umsturz"-Begriff, der sowohl Putsch als auch Revolution bedeuten kann, wurde in einem weiteren Artikel angeführt, um die Militäraktion der G30S zum Auftakt eines weitreichenden bewaffneten Aufstands zu stilisieren:103 Die "Revolutionsgarden [trainierten] im geheimen"104, schreibt Der Spiegel, während die PKI-Jugendorganisation "mit eingeschmuggelten chinesischen Ysung-Karabinern" ausgestattet und die Streitkräfte infiltriert worden seien. Listen mit „60.000 Todeskandidaten“ habe die Armee "angeblich" gefunden, ebenso wie "Folterwerkzeuge - etwa zum fachgemäßen Herausquetschen der Augen - und Handgranaten [...], mit denen KP-Funktionäre ihre Nachbarschaft von unsicheren Elementen säubern sollten". ${ }^{105}$

Das Folter-Narrativ war zentral für die Aufhetzung der indonesischen Bevölkerung und zur Legitimierung der Vernichtungsmaßnahmen, die durch die Schilderung der Brutalität (in Zusammenhang mit dem Umsturz-Narrativ) als Notwehr- oder Racheakte verständlich gemacht werden konnten. Die meisten der Spiegel-Artikel sind von

100 Zum Zusammenspiel von militärischer Kampagne und Massenbeteiligung siehe Gerlach, Extremely Violent Societies, S. 17-91.

101 O. A., Der Spiegel, 27.10.1965, S. 32.

102 O. A., Der Spiegel, 3.1.1966, S. 58.

103 In einem Interview mit dem Spiegel spricht Suharto 1970 von einem Aufstand mit tausenden (noch lebenden) Beteiligten: „Wir trennen die Inhaftierten in drei Kategorien: A, B und C. Die Gruppe A war tatsächlich am Putsch beteiligt. Das sind etwa 5.000 Häftlinge. Gruppe B nahm ebenfalls am Aufstand teil, das Beweismaterial reicht aber nicht aus. Hier handelt es sich um etwa 15.000. Gruppe $C$ sind die Verdächtigen, rund 22.000. Bei weiteren 16.000 Häftlingen ist der Grad der Beteiligung am Umsturzversuch ungeklärt; ihre Gruppenzugehörigkeit ist noch nicht bestimmt. Darunter befinden sich 4.000 Angehörige der Streitkräfte. Insgesamt sind das 58.000 politische Gefangene." (O. A., „Wir haben 58.000 politische Gefangene“, in: Der Spiegel, Nr. 27, 29.6.1970, S. 97-98, hier S. 97, [http://www.spiegel.de/spiegel/print/d-44931081.html], eingesehen 11.8.1970).

104 O. A., Prächtiger Kerl, in: Der Spiegel, Nr. 42, 13.10.1965, S. 114-116, hier 116, [http://www.spiegel.de/spiegel/ print/d-46274703.html], eingesehen 11.8.2017.

105 O. A., Der Spiegel, 27.10.1965, S. 32. 
diesen beiden Topoi - Folter und Rachemotiv - geprägt. ${ }^{106}$ Bereits im Oktober wurde von den „bis zur Unkenntlichkeit verstümmelten Leichen“107 der Generäle berichtet, in zwei weiteren Artikeln wird dies näher ausgeführt: Die Leichen waren fürchterlich verstümmelt. Einem Toten hatte man das abgeschnittene Geschlechtsteil in den Mund gesteckt. [...] Angeblich hatten Mitglieder der kommunistischen Frauen-Organisation Gerwani das Gemetzel auf dem Gewissen." ${ }^{108}$ Die Einschreibung von einfachen PKIMitgliedern in die Erzählung der Tat, den brutalen Mord, macht die maßlose Reaktion für die Leser_innenschaft nachvollziehbar und lässt sie bis zu einem gewissen Grad legitim erscheinen. Die Hervorhebung der Frauen bei der Folterszene, wie sie im offiziellen Narrativ durchgängig anzutreffen ist, bedient nicht nur sexistische und sexistischantikommunistische Topoi („die rote Furie“), sie zementiert zudem die These der PKIKollektivschuld.

Aussagekräftig ist auch die folgende Passage des Artikels: „Die Rache der Antikommunisten“, so der Autor, "war nicht minder grausam."109 Durch diese Formulierung wird - ebenso wie mit den Begriffen "Gegencoup" und "Gegenterror" - eine Parallele gezogen, die die Aktionen der Konfliktparteien auf dieselbe moralische Stufe hebt. Die Beschreibung der Vorgänge als "Racheakte", 10 für die stellenweise auch Verständnis gezeigt wird (die Armee, "die durch Mordanschläge [...] sechs ihrer höchsten Generäle verloren hatte, machte auch selbst Jagd auf die Roten"111) suggeriert Spontanität und Wutausbruch und deutet die systematisch-planvolle Vernichtung ganzer Bevölkerungsteile zum Resultat einer singulären, pogromartigen Aktion um. Dieselbe Funktion erfüllt die Wiedergabe eines angeblich verhinderten Anschlags auf ein TrinkwasserReservoir durch die PKI, "wodurch die Erbitterung gegen die Kommunisten“112 groß geworden sei.

Die Sichtweise, die durch diese Darstellung vermittelt wird, ist primär die von außen

106 In fünf von elf Artikeln finden sich Erwähnungen oder Beschreibungen der Folterungen, in vier von elf explizite Erwähnungen des Rachemotivs.

107 O. A., Der Spiegel, 13.10.1965, S. 117

108 O. A., Der Spiegel, 9.5.1966, S. 99. Die ausgeschmückteste Variante dieses Narrativs liefert Vittachi:„Eines ist jedenfalls sicher: Die Generäle wurden in der brutalsten Weise verstümmelt. Eine der Beteiligten an den Ereignissen jener Nacht, Frau Djamilah, die 15-jährige Ehefrau eines Kommunisten, erzählte, was sich zutrug: ,Kleine Messer und Rasierklingen wurden verteilt. Ich bekam nur eine Rasierklinge. Von weitem sahen wir einen untersetzten Mann im Schlafanzug, die Hände mit einem roten Tuch gefesselt, die Augen mit einem roten Tuch verbunden. Ein Zugführer befahl uns, diese Person zu verprügeln und ihm dann seine Männlichkeit abzuschneiden. Herr und Frau Sastro, die Führer unserer Ortsgruppe Tandjung Priok, fingen als erste an zu schlagen und zu schneiden. Dann folgten andere Genossen ... Schließlich beteiligte auch ich mich an der Quälerei. Alle 100 Frauen taten es und sahen zu ... Dreimal wurde auf das Opfer geschossen, dann fiel es hin, war aber noch nicht tot. Ein Mann in grüner Uniform mit gebogenen weißen Schulterstücken gab die Befehle und stach selbst auf das Opfer ein bis es starb." (O. A., Der Spiegel, 27.2.1967, S. 110.)

109 O. A., Der Spiegel, 9.5.1966, S. 99.

110 Im Oktober 1965: „Angreifer waren antikommunistische Moslems, die in den beiden letzten Wochen Rache für den mißglückten, von den Kommunisten inspirierten Putsch vom 30. September nahmen." (O. A., Der Spiegel, 27.10.1965, S. 32). Und im Oktober 1966: „Die neuen Militär-Herren Indonesiens beschuldigen Peking der Urheberschaft an dem - mißglückten - kommunistischen Putsch vom September 1965. Ihre Rache traf die Gelben im Lande." (O. A., Gefährliche Farbe, in: Der Spiegel, Nr. 44, 24.10.1966, S. 132-133, hier 133, [http://www. spiegel.de/spiegel/print/d-46414849.html], eingesehen 11.8.2017.)

111 O. A., Der Spiegel, 27.10.1965, S. 32.

112 O. A., Der Spiegel, 9.5.1966, S. 100. Diese Passage evoziert den antisemitischen Topos der Brunnenvergiftung; die Konstruktion von „jüdisch-marxistischen" Feindbildern hat im Nationalismus eine lange Tradition. 
(des "Westens"/der BRD) auf die Ereignisse im Land. Betont werden die Implikationen für das weltpolitische Machtgefüge, selbst im Augenzeugenbericht schildert ein „deutscher Doktorand, der für das Goethe-Institut in Bangkok tätig war ${ }^{\prime \prime 113}$, seine Eindrücke. Sie werden zwar als innerindonesische Angelegenheit verhandelt, dies jedoch in reduktionistischer Terminologie ("die Kommunist_innen“" die Chines_innen“) ohne Berücksichtigung der Spezifika und Heterogenität des Landes. Gleichzeitig wird der Blick von oben (Fokus auf das politische System und die Elite, vor allem auf die Person Sukarnos inklusive Ehe- und Privatleben; vom Massaker Betroffene oder Täter_innen aus der Bevölkerung bleiben anonym und kommen nicht zu Wort) und rechts (die unkritische Übernahme des Narrativs der Suharto-Militärs, die Parallelisierung der Massaker und Verfolgungen mit der angeblichen Brutalität der „Roten“) favorisiert. Ausgeblendet wird dadurch die Perspektive jener Personengruppen, für die die Ereignisse die drastischsten Konsequenzen hatten: einfache Parteimitglieder, Sympathisant_innen und ihre Angehörigen. ${ }^{114}$

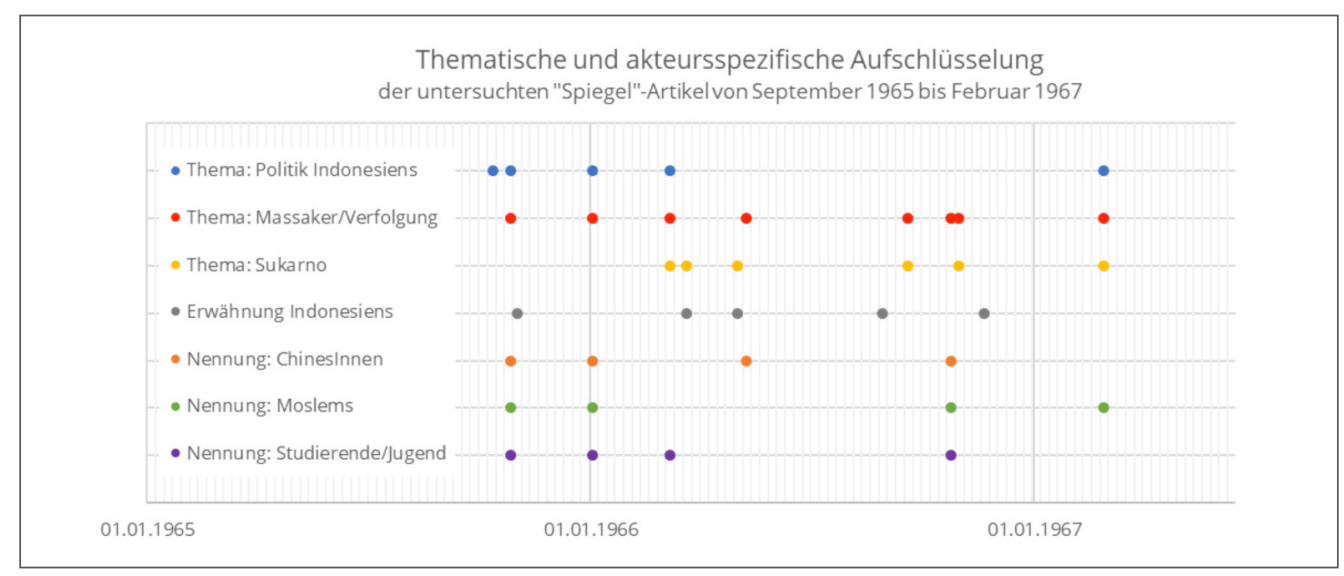

Abbildung 2: Analyse der Spiegel-Berichterstattung nach dem 1. Oktober bis zur Machtübernahme Suhartos ${ }^{115}$

\section{Persistenz und Dekonstruktion des master narrative ab 1998}

Die Existenz eines hegemonialen Narrativs impliziert die Existenz von subversiven bzw. Gegennarrativen, gegen die sich das master narrative durchgesetzt hat und behaupten musste. Auch bei der polizeilich-gewaltförmigen Durchsetzung eines Narrativs wie unter dem New-Order-Regime bestehen Möglichkeiten für Gegen-Erinnerungspraxen. Durch die Verdrängung aus dem öffentlichen Raum bleiben diese Kulturen der Erinnerung vielfach prekär und sind auf marginalisierte Kanäle der kommunikativen Reproduktion angewiesen: Alternative Einschätzungen der G30S, ebenso wie die Erfahrungen der Opfer der Gewalt sind aus dem Feld des öffentlich Sagbaren verdrängt, werden durch die Zirkulation verbotener Literatur und Filme ebenso wie durch münd-

113 O. A., Der Spiegel, 9.5.1966, S. 99.

114 Die systematischen Vergewaltigungen finden in keinem Artikel Erwähnung.

115 Die vier unteren Analysekategorien geben das Hauptthema des Artikels an bzw. ob die Ereignisse von $1965 / 66$ nur in einer Textpassage erwähnt werden; bei Thematisierung der Massaker und Verfolgungen ist in der Grafik zudem ersichtlich, welche der drei obenstehenden Opfer-/Täter_innen-Gruppen angesprochen werden. 
liche Erzählungen innerhalb von Familien, Freundeskreisen und Dorfgemeinschaften weitergegeben. Sie erfüllen aufgrund der fortwährenden Repression und Stigmatisierung der Nachfahren von ehemaligen Inhaftierten und Verdächtigten bis in die dritte Generation eine zentrale Funktion im Identifikationsprozess und in der Selbstwahrnehmung der Betroffenen: ${ }^{116}$

„Any public expression that implied doubt in the validity of the official history of 1965 or made reverence to alternative narratives among scholars overseas was declared against the law and was liable to criminal prosecution. Notwithstanding such intimidation, dissenting voices emerged from time to time during the New Order rule, if only to be immediately suppressed."117

Mit dem Sturz Suhartos 1998 veränderten sich die diskursiven Bedingungen massiv, da die zentrale Funktion des Narrativs zur Legitimierung der Herrschaft obsolet wurde, obschon die Aufarbeitung der Massaker auf den Widerstand unterschiedlicher, von Kontinuität geprägter Elitenfraktionen stößt. Die Herausforderung besteht zum einen in der Organisierung und Ausarbeitung alternativer Narrative und diskursiver Frameworks und zum anderen ihrer Durchsetzung gegenüber den bestehenden. Diese zielen auf die Anerkennung der Unrechtmäßigkeit der Morde, Verfolgungen und Repressionen und stellen das PKI-Putsch-Narrativ in Frage. ${ }^{118}$ Auf zivilgesellschaftlicher Ebene hat die Phase ab 1998 (in Indonesien „Reformasi” genannt) zur Gründung zahlreicher Menschenrechts- und Rechtshilfeorganisationen sowie Opfervereinigungen geführt. Auch im Pressewesen und Wissenschaftsbetrieb sehen sich viele der Aufklärung der Ereignisse verpflichtet. ${ }^{119}$ Unter den staatlichen Institutionen ist die bereits unter Suharto initiierte nationale Menschenrechtskommission Komnas HAM zum wichtigsten Akteur im Aufklärungsprozess geworden. Der Vorstoß von Abdurrahman Wahid, Vorsitzender der islamischen Nahdlatul-Ulama-Organisation (1984-1999) und Staatspräsident von 1999 bis 2001 sowie die Bemühungen von Megawati Sukarnoputri, Tochter Sukarnos, ehemalige Staatspräsidentin (2001-2004) und Vorsitzende der linksliberalen PDI-P-Partei (seit 1998) lassen sich als Beispiele für den Veränderungsprozess im Parteiensystem anführen. ${ }^{120}$

Andererseits erklärte der gegenwärtige Präsident Joko Widodo (seit 2014 im Amt, PDI-P-Partei; von der ausländischen Presse wurde er als „Obama Indonesiens" betitelt) im Jahr 2015 im Rahmen der Pankasilia-Sakti-Gedenkveranstaltung in Lubang Buaya, der er in allen drei seiner bisherigen Amtsjahre beigewohnt hatte, er hoffe, "the G30S/ PKI [coup] will not happen again in our beloved country" und er hätte bezüglich der Massaker 1965/66 „no thoughts about apologizing.“121 Die Debatte um eine offizielle

116 Heryanto, State Terrorism, S. 159-195.

117 Ariel Heryanto, Identity and Pleasure. The Politics of Indonesian Screen Culture, Singapore 2014, S. 82.

118 Der Ablauf der Ereignisse vom 1. Oktober ebenso wie die grundsätzliche Charakterisierung der PKI und linker Bewegungen durch das Regime werden weniger thematisiert.

119 Ufen, Vergangenheitspolitik, S. 6-7; siehe auch Keller, Indonesien 1965ff., S. 47-58.

120 Ufen, Vergangenheitspolitik, S. 5-6.

121 Ina Parlina/Fedina S. Sundaryani, Jokowi rejects apology, promotes stability, in: The Jakarta Post, 2.10.2015, [http://www.thejakartapost.com/news/2015/10/02/jokowi-rejects-apology-promotes-stability.html], eingesehen 15.8.2017. 
Entschuldigung und Anerkennung der Unrechtmäßigkeit der Massaker erreichte im Sommer 2016 mit der Publikation des Endberichts des International People's Tribunal on 1965 Crimes Against Humanity in Indonesia (IPT 1965), eines international besetzten Panels von Richter_innen, einen vorläufigen Höhepunkt. Dieser kam zum Schluss, in den Jahren 1965/66 seien unterschiedliche Verbrechen gegen die Menschlichkeit begangen worden, für die der indonesische Staat verantwortlich gemacht werden muss. ${ }^{122}$ Bezüglich der Ereignisse heißt es dort: „On the night of 30 September 1965, a small group of pro-Sukarno army officers with support of the PKI leader launched an action against anti-Sukarno officers - six generals and one lieutenant were killed."123 Die Regierung folgte der IPT-Empfehlung nach einer offiziellen Anerkennung nicht. Im Vorfeld war eine kontrovers geführte Debatte entbrannt, muslimische und andere Gruppen hatten sich gegen eine Entschuldigung ausgesprochen. ${ }^{124}$

\subsection{Die Rezeption im deutschsprachigen Raum im Wandel (Der Spiegel-Analyse 2)}

Zur Beantwortung der Frage, ob und inwieweit sich der mediale Diskurs zu 1965/66 im deutschsprachigen Raum nach dem Ende des Suharto-Regimes 1998 verändert hat, soll im Folgenden die Darstellung der Ereignisse im Spiegel ab dem Jahr 1990 analysiert werden. Bis August 2017 finden sich 14 Artikel dazu, im Gegensatz zum Korpus aus den 1960er-Jahren beschäftigen sich die meisten (zwölf) lediglich am Rande bzw. in kurzen Textpassagen damit. ${ }^{125}$

Anfang der 1990er-Jahre wird erklärt, 1965 hätten "die Kommunisten in Jakarta [geputscht] ", "mehr als eine halbe Million Menschen“ sei getötet worden. ${ }^{126}$ In den Artikeln von 1996 werden zwar noch keine Zweifel am offiziellen Narrativ des New-Order-Regimes artikuliert, die Formulierungen stellen die Tatsache des PKI-Putsches jedoch als Meinung des Militärs dar: „1965“, heißt es dort, "hatte die Armeeführung unter Generalmajor Suharto die Kommunistische Partei Indonesiens (PKI) für einen gescheiterten Putsch verantwortlich gemacht."127 Ein völlig anderes Bild vermittelt der Autor der Passage eines Artikels zwei Jahre später, die besagt, „damals [seien] bei Unruhen, die von den Kommunisten geschürt wurden, rund 500.000 Menschen [gestorben]"128, wo-

122 Yenni Kwok, Indonesia's Mass Killings of 1965 Were Crimes Against Humanity, International Judges Say, in: Time, 20.7.2016, [http://time.com/4414438/indonesia-crimes-against-humanity-1965/], eingesehen 15.8.2017. Siehe auch International People's Tribunal on 1965 Crimes Against Humanity in Indonesia, Final Report of the IPT 1965: Findings and Documents of the IPT 1965, O. D., [http://www.tribunal1965.org/en/final-report-of-the-ipt-1965/], eingesehen 15.8.2017.

123 International People's Tribunal, Final Report.

124 O. A., Hard-line groups to protest 1965 apology at State Palace, in: The Jakarta Post, 2.6.2016, [http://www. thejakartapost.com/news/2016/06/02/hard-line-groups-to-protest-1965-apology-at-state-palace.html], eingesehen 15.8.2017.

125 Spiegel-Suche, Suchmaskeneingabe "indonesi*“ UND "1965"/,indonesi*" UND "putsch*"/,indonesi*" UND „massaker", Zeitraum „alles", „Volltext”, in: Der Spiegel, [http://www.spiegel.de/suche/index.html], abgefragt 3.8.2017.

126 O. A., „Unsere Gedanken sind tot“, in: Der Spiegel, Nr. 17, 1.7.1990, S. 130-133, hier S. 132, [http://www.spiegel.de/ spiegel/print/d-13487469.html], eingesehen 11.8.2017.

127 O. A., Rotes Gespenst, in: Der Spiegel, Nr. 35, 26.8.1996, S. 129, [http://www.spiegel.de/spiegel/print/d-9086109. html], eingesehen 11.8.2017; O. A., Die Insel der Friedhöfe, in: Der Spiegel, Nr. 43, 21.10.1996, S. 179-187, hier S. 182, [http://www.spiegel.de/spiegel/print/d-9108473.html], eingesehen 11.8.2017.

128 Christoph Pauly, 'Wir brauchen mehr als Aspirin“, in: Der Spiegel, Nr. 4, 19.1.1998, S. 82-83, hier S. 83. [http://www. 
durch die PKI auch für die Massaker selbst verantwortlich gemacht wird. In den drei darauffolgenden Artikeln, die sich mit den Mai-Unruhen 1998 und den ersten Wahlen 1999 befassen, ist hingegen wieder von einem „angeblich kommunistischen Putschversuch" die Rede. ${ }^{29}$ In Nasutions Nachruf aus dem Jahr 2000 werden die Ereignisse von 1965/66 als „ein[...] Putschversuch“130 bezeichnet, während der auf Suharto acht Jahre später ein recht differenziertes Bild der Situation wiedergibt: „[A]ngeblich wollten die Genossen gegen das rechte Militär die Macht ergreifen. In Folge starben zwischen 500.000 und eine Million Linke, Gewerkschaftler und Indonesier chinesischer Abstammung bei Massakern, angezettelt von Suhartos Elitetruppen und ausgeführt von einem blutrünstigen Mob."131

Erst in den Jahren 2013 und 2014 beschäftigen sich zwei Spiegel-Artikel hauptsächlich mit den Ereignissen von 1965/66, anlässlich der Uraufführung von Joshua Oppenheimers Dokumentarfilm „The Act of Killing“ in Jakarta. Dieser dokumentiere, so der Artikel aus dem KulturSpiegel,

„die Massenmorde an Kommunisten und anderen vermeintlichen Staatsfeinden während des Militärputsches in Indonesien in den sechziger Jahren - erdacht von General Suharto, ausgeführt unter anderem von den willigen Helfern der Pancasila-Jugend".132

Die Opferzahl wird mit „mehr als eine[r] Million“ angegeben, es wird darauf verwiesen, dass "[i]n einem staatlichen Propagandafilm aus den achtziger Jahren [...] die gesamte Schuld an den Massenmorden dem Putschversuch der Kommunisten"133 gegeben wurde, wodurch der Artikel wiederum das Narrativ des PKI-Putschs bedient, die Partei jedoch im gesamten Text nicht erwähnt. Zudem wird die Opfergruppe der Chines_innen hervorgehoben, deren im Film gezeigte Verfolgung „eine Diskussion über Menschenrechtsverletzungen in Gang setzte, die unabhängig von der kommunistischen Frage ist."134 Im Kurzbericht über die Ausstrahlung des Films im bundesdeutschen Fernsehen vom März 2014 ${ }^{135}$ wird die Frage der Urheberschaft des Putschs ausgeklammert, ebenso wie in einem Artikel vom nächsten Jahr. ${ }^{136}$ Der letzte Artikel des Spiegel-

spiegel.de/spiegel/print/d-7810108.html], eingesehen 11.8.2017.

129 O. A., Der sterbende Elefant, in: Der Spiegel, Nr. 21, 18.5.1998, S. 166-169, hier S. 169, [http://www.spiegel.de/ spiegel/print/d-7892910.html], eingesehen 11.8.2017; O. A., Sturz in die Finsternis, in: Der Spiegel, Nr. 50, 7.12.1998, S. 200-202, hier S. 202, [http://www.spiegel.de/spiegel/print/d-8440705.html], eingesehen 11.8.2017; O. A., ,Wie geht's dir, alte Tante Java?", in: Der Spiegel, Nr. 23, 7.6.1999, S. 186-187, hier S. 186, [http://www.spiegel.de/spiegel/ print/d-13667566.html], eingesehen 11.8.2017.

130 O. A., Abdul Haris Nasution, in: Der Spiegel, Nr. 39, 25.9.2000, S. 310, [http://www.spiegel.de/spiegel/ print/d-17436657.html], eingesehen 11.8.2017.

131 O. A., Suharto, in: Der Spiegel, Nr. 6, 2.2.2008, S. 146, [http://www.spiegel.de/spiegel/print/d-55668252.html], eingesehen 11.8.2017.

132 Ayu Utami, Sturz der Henker, in: KulturSpiegel, Nr. 11, 28.10.2013, o. S., [http://www.spiegel.de/spiegel/ kulturspiegel/d-117763264.html], eingesehen 11.8.2017.

133 Utami, KulturSpiegel, 28.10.2013, o. S.

$134 \mathrm{Ebd}$

135 O. A., Joshua Oppenheimer, in: Der Spiegel, Nr. 10, 1.3.2014, S. 136, [http://www.spiegel.de/spiegel/ print/d-125300729.html], eingesehen 11.8.2017.

136 O. A., Der Triumph der Killer, Interview mit Klaus Theweleit, in: Der Spiegel, Nr. 20, 9.5.2015, S. 136-139, hier 137, [http://www.spiegel.de/spiegel/print/d-134879030.html], eingesehen 11.8.2017. 
Korpus ist mit August 2015 datiert, auch dieser, ein Landesportrait, thematisiert die Ereignisse von 1965/66:

„Vor allem interessierten sie [eine indonesische Schriftstellerin] die Massaker an den Kommunisten und Linksliberalen aus den Jahren 1965/66. Hunderttausende 'Volksschädlinge' wurden damals nach einem gescheiterten Putsch gegen den Militärdiktator Sukarno hingerichtet oder in Lager verschleppt."137

Und weiter heißt es:

"An den Pogromen vor 50 Jahren haben sich nicht nur Militärs und Polizisten beteiligt, sondern auch islamische Organisationen, deren Mitglieder durch die Nachbarschaft zogen und 'Verräter' aus den Häusern zerrten." ${ }^{38}$

Zusammenfassend lässt sich in diesem Spiegel-Korpus eine deutliche Diskursverschiebung im Zeitraum von 1990 bis 2017 ausmachen: Während am Anfang der 90er-Jahre das Narrativ des PKI-Putschs reproduziert wurde, wird dies in der zweiten Hälfte des Jahrzehnts - vor und nach dem Sturz Suhartos (1996 bis 2000), also in jener Phase, in der eine zahlenmäßige Häufung in der Berichterstattung auszumachen ist - relativiert, in der Regel durch den Zusatz „angeblich“.

In den untersuchten Textpassagen ist zudem eine zweite Verschiebung durch die Rezeption des Dokumentarfilms von Joshua Oppenheimer nach 2013 auszumachen: Die Verkettung Putsch $\rightarrow$ Massaker wird umgedreht und tendenziell aufgelöst. Die Beschäftigung mit den Massakern und Verfolgungen steht nicht mehr im Schatten der Aktionen der G30S, als deren Konsequenz sie gesehen wurden, wodurch dieses von vielen schon immer als unzureichend gesehene Deutungsangebot wegbricht und die Frage nach dem Wie und Warum zum ersten Mal offen artikuliert werden kann; der Putsch wird zwar erwähnt, eine Zuschreibung jedoch unterlassen, da diese für die Aufarbeitung der Massaker nur bedingt von Relevanz ist. Die Ermordung der sechs Generäle, Kernelement des PKI-Putsch-Narrativs, findet lediglich 1990 im ersten Artikel des untersuchten Spiegel-Korpus Erwähnung.

Im Vergleich mit der Darstellungsweise in den 1960er-Jahren ist festzustellen, dass mit der Diskurs- auch eine Perspektivenverschiebung einhergeht. Es wird versucht, die indonesische Gesellschaftsrealität in ihrer Komplexität erfahrbar zu machen und neben den politischen auch die sozialen Verhältnisse zu berücksichtigen. Exemplarisch ist hierbei der Artikel "Wie geht's dir, alte Tante Java” von 1999, in dem Personen verschiedener politischer Lager und Klassen zu Wort kommen. ${ }^{139}$ Vor allem aber - und das muss als Verdienst Oppenheimers gesehen werden - rückt nunmehr die Perspektive der Täter_innen (und Opfer, auch wenn sein zweiter Film „The Look of Silence“ von

137 O. A., Das Reich des sanften Islam, in: Der Spiegel, Nr. 32, 1.8.2015, S. 80-83, hier 83, [http://www.spiegel.de/ spiegel/print/d-137878522.html], eingesehen 11.8.2017.

138 O. A, Der Spiegel, 1.8.2015, S. 83.

139 O. A., Der Spiegel, 7.6.1999, S. 186-187. 
$2015^{140}$ in der Print-Version des Spiegel unerwähnt bleibt) der Massaker in den Vordergrund.

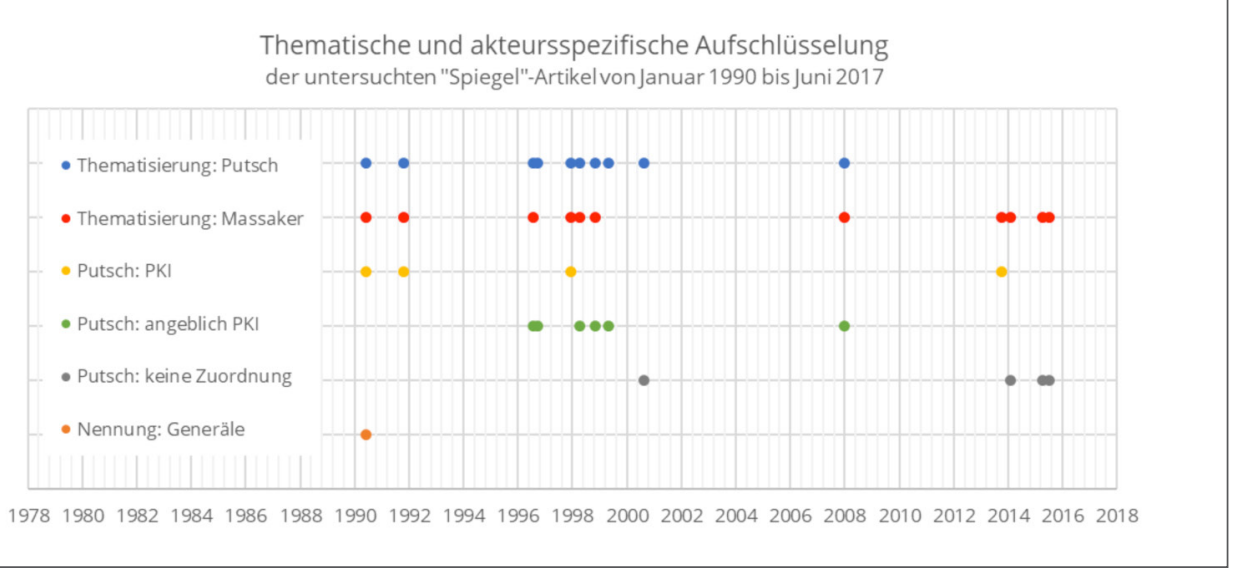

Abbildung 3: Analyse der Spiegel-Berichterstattung ab 1990141

\section{Schluss: Veränderte Diskurse, veränderte Perspektive}

Auf den vorangegangenen Seiten wurde gezeigt, dass die Ereignisse von 1965/66 (Aktionen der G30S, Machtergreifung Suhartos, Massaker und Verfolgungen) im Kontext der welt- und innenpolitischen Machtkämpfe zu verstehen und immer noch Gegenstand kontroverser Debatten sind. Die These eines PKI-Putschs, bei der die Partei als Gesamtorganisation eine Machtübernahme in Indonesien geplant habe, gilt in unabhängigen zeitgenössischen und neueren Forschungsarbeiten als weitgehend widerlegt. Sie ist Kern der Narration, mit der die Militärs um Suharto unmittelbar nach den Ereignissen Anfang Oktober 1965 eine Vernichtungskampagne gegen die PKI initiierten und die sie nach der Machtergreifung 1966/67 als master narrative mit der gleichzeitigen Funktion der Mobilisierung und Passivierung (im Zuge der Herrschaftssicherung und Unterdrückung von Widerspruch) durchsetzten. Wesentliches Element des Narrativs, das in allen Dimensionen der kollektiven Erinnerung institutionalisiert wird, ist die Verkettung der Ereignisse des 1. Oktober mit Putsch und Revolution sowie die Gleichsetzung von G30S und PKI.

Im Museum Pengkhianatan PKI, Teil des gewichtigsten Erinnerungsortes zu 1965/66, wird dies erreicht, indem eine direkte Verbindungslinie zwischen den gewalttätigen Aktionsformen von Kommunist_innen in den 1940ern (insbesondere den Maidun-Ereignissen 1948) und den G30S-Aktionen gezogen wird: Der PKI-Partei, einseitig diffamierend charakterisiert als gewalttätig, umstürzlerisch und antinational, steht das Mili-

140 Joshua Oppenheimer/Signe Byrge Sørensen, The Look of Silence, DVD, 103 min., Dänemark 2014. In diesem Film vollzieht Oppenheimer einen Perspektivenwechsel, nach den Täter_innen in „The Act of Killing“ kommen nun die Opfer der Massaker und Verfolgungen zu Wort.

141 Die vier unteren Analysekategorien geben das Hauptthema des Artikels an bzw. ob die Ereignisse von 1965/66 nur in einer Textpassage erwähnt werden; bei Thematisierung der Massaker und Verfolgungen ist in der Grafik zudem ersichtlich, welche der drei obenstehenden Opfer-/Täter_innen-Gruppen angesprochen werden. 
tär gegenüber, Verteidiger von Recht, Ordnung und Nation. Dieselbe Funktion erfüllen die erfundenen Folterszenen, durch die die Brutalität und Mitschuld der PKI-Mitglieder verdeutlicht werden sollen. Die Ereignisse werden im Spiegel im Zeitraum von Oktober 1965 bis März 1967 durchaus thematisiert. Die Massaker und Verfolgungen finden schon früh (ab Ende Oktober) Beachtung, werden jedoch primär als spontane (Re-) Aktionen der Bevölkerung erklärt, wodurch Suharto und das Militär entlastet werden. Die Hervorhebung von Chines_innen bzw. Muslim_innen und Jugendlichen bei den Opfer- und Täter_innengruppen erfüllt eine entpolitisierende Funktion und macht die Massaker für die westliche Leser_innenschaft in einem reduktionistischen Framework lesbar. Das Narrativ des PKI-Putschs wird vom Spiegel durchgängig, d. h. in allen wesentlichen Bestandteilen und in allen Artikeln, übernommen: Verschwörung der PKI, Umsturzversuch, Folter und Misshandlung der Generäle, Racheaktionen der Bevölkerung. Gerüchte mit diffamierendem Charakter (Brunnenanschlag) finden sich in den Artikeln, eine kritische Positionierung wird nicht versucht. Es dominiert die Perspektive von außen ("westlich"), oben (elitenbezogen) und rechts (nationalistisch).

Im Zeitraum ab 1998, nach dem Sturz des Suharto-Regimes, findet in der indonesischen Gesellschaft ein erinnerungskultureller Wandel statt. Zum ersten Mal können alternative Narrationen offen artikuliert und teilweise im kulturellen Gedächtnis verankert werden, auch wenn das PKI-Putsch-Narrativ noch immer prägend und im politischen System verankert ist. Ab den 90ern kann auch im Spiegel eine Diskursund Perspektivenverschiebung ausgemacht werden. Die Urheberschaft der PKI am „Putsch" wird angezweifelt, der Fokus verschiebt sich von diesem auf die Massaker und Verfolgungen. Dadurch wird zum einen der komplexen Lebensrealität in Indonesien vermehrt Rechnung getragen, zum anderen die vormals ausgeklammerte Perspektive der Opfer und Täter_innen zur Sprache gebracht.

Weitere Forschungsarbeiten werden diese ersten Befunde durch eine räumliche und zeitliche Ausweitung des Analysefeldes vertiefen müssen, etwa durch Einbeziehung weiterer Medien, insbesondere aus anderen soziopolitischen Spektren bzw. Ländern. Zudem wäre für die Zeit nach 1990 die genauere Betrachtung der Jahrestage 1995, 2005 und 2015 aufschlussreich. Dies wäre für die Bewusstmachung der Bedeutung eines kritischen Umgangs mit Medien und Meistererzählungen dienlich und kann als Beitrag zur Schaffung anderer - um in der Terminologie Andersons zu bleiben - Erfindungen der Geschichte und damit einer anderen Erinnerungskultur verstanden werden.

\section{Literatur}

Abdullah, Anzar, Contemporary History of Indonesia between Historical Truth and Group Purpose, in: Review of European Studies 7 (2015), Heft 12, S. 179-185.

Anderson, Benedict/McVey, Ruth, A Preliminary Analysis of the October 1, 1965, Coup in Indonesia, Singapur 2009.

Anderson, Benedict, Imagined Communities. Reflections on the Origin and Spread of Nationalism, London-New York 2006. 
Assmann, Aleida, Erinnerungsräume. Formen und Wandel des kulturellen Gedächtnisses, München 1999.

Assmann, Jan, Das kulturelle Gedächtnis. Schrift, Erinnerung und politische Identität in frühen Hochkulturen, München 2000.

Becker, Florian, Gramsci lesen. Einstieg in die Gefängnishefte, Hamburg 2014.

Bhabha, Homi K. (Hrsg.), Nation and Narration, London-New York 1990.

Bunnell, Frederick, American "Low Posture" Policy toward Indonesia in the Months Leading up to the 1965 "Coup", in: Indonesia 25 (1990), Heft 50, S. 29-60.

Christie, Clive J., Ideology and Revolution in Southeast Asia 1900-1980. Political Ideas of the Anti-Colonial Era, Richmond 2001.

Choppel, Charles A., Preface, in: Charles A. Choppel (Hrsg.), Violent Conflicts in Indonesia. Analysis, Representation, Resolution, London-New York 2006, S. XV-XVIII.

Choppel, Charles A., Anti-Chinese Violence in Indonesia after Soeharto, in: Leo Suryadinata (Hrsg.), Ethnic Chinese in Contemporary Indonesia, Singapore 2008, S. 117-136.

Cribb, Robert/Coppel, Charles, A Genocide That Never Was: Explaining the Myth of Anti-Chinese Massacres in Indonesia, 1965-66, Journal of Genocide Research 11 (2009), Heft 4, S. 219-239.

Crouch, Harold, The Army and Politics in Indonesia, Singapore 2007.

Dwyer, Leslie/Degung Santikarma „When the World Turned to Chaos”. 1965 and Its Aftermath in Bali, Indonesia, in: Robert Gellately/Ben Kiernan (Hrsg.), The Specter of Genocide. Mass Murder in Historical Perspective, Cambridge 2003, S. 289-306.

Fionna, Ulla, The Institutionalisation of Political Parties in Post-authoritarian Indonesia. From the Grass-roots Up, Amsterdam 2013.

Gavi, Philippe, Konterrevolution in Indonesien, Frankfurt a. M. 1969.

Gerlach, Christian, Extremely Violent Societies. Mass Violence in the Twentieth-Century World, Cambridge 2010.

Halbwachs, Maurice, Das kollektive Gedächtnis, Frankfurt a. M. 1991.

Hendrikx, Paula, "1965" in Indonesia Today. The Politics of Memory in Post-Suharto Indonesia and the Perceptions of the 1965-66 Past among High School Students in Yogyakarta in 2016, Dipl. phil. Leiden 2017.

Heryanto, Ariel, State Terrorism and Political Identity in Indonesia. Fatally Belonging, London-New York 2006.

Heryanto, Ariel, Identity and Pleasure. The Politics of Indonesian Screen Culture, Singapore 2014. 
International People's Tribunal on 1965 Crimes Against Humanity in Indonesia, Final Report of the IPT 1965: Findings and Documents of the IPT 1965, o. D. [http://www. tribunal1965.org/en/final-report-of-the-ipt-1965/, eingesehen 15.8.2017.

Jarausch, Konrad H./Sabrow, Martin, "Meistererzählung". Zur Karriere eines Begriffs, in: Konrad H. Jarausch/Martin Sabrow (Hrsg.), Die historische Meistererzählung. Deutungslinien der deutschen Nationalgeschichte nach 1945, Göttingen 2002.

Keller, Anett (Hrsg.), Indonesien 1965ff. Die Gegenwart eines Massenmordes. Ein politisches Lesebuch, Berlin 2015.

Klein, Naomi, The Shock Doctrine. The Rise of Disaster Capitalism, New York 2007.

Kwok, Yenni, Indonesia's Mass Killings of 1965 Were Crimes Against Humanity, International Judges Say, in: Time, 20.7.2016, [http://time.com/4414438/indonesia-crimesagainst-humanity-1965/], eingesehen 15.8.2017.

Leksana, Grace, Reconciliation Through History Education: Reconstructing the Social Memory of the 1965-66 Violence in Indonesia, in: Birgit Bräuchler (Hrsg.), Reconciling Indonesia. Grassroots Agency for Peace, London-New York 2009, S. 175-191.

Lyotard, Jean Francois, Das postmoderne Wissen. Ein Bericht, Graz 1986.

Markas Besar Tentar Nasional Indonesia Pusat Sejarah/Headquarters of the Indonesian National Military Center for TNI History (Hrsg.), Buku Panduan Monumen Pancasila Sacti/The Guide Book of Pancasila Sakti Monument, Jakarta 2008.

Melvin, Jess, Why Not Genocide? Anti-Chinese Violence in Aceh, 1965-1966, in: Journal of Current Southeast Asian Affairs 3 (2013), Heft 32, S. 63-91.

Melvin, Jess, Documenting Genocide, in: Inside Indonesia 32 (2015), Heft 122, [http:// www.insideindonesia.org/documenting-genocide-3], eingesehen 10.8.2017.

McGregor, Katharine E., History in Uniform. Military Ideology and the Construction of Indonesia's Past, Singapore 2007.

Notosusanto, Nugroho/Saleh, Ismail, The Coup Attempt of the September 30 Movement in Indonesia, Jakarta 1968.

Notosusanto, Nugroho/Poesponegoro, Marwati Djoned u. a. (Hrsg.), Sejarah Nasional Indonesia, Jakarta 1975.

Neubert, Harald, Antonio Gramsci: Hegemonie - Zivilgesellschaft - Partei. Eine Einführung, Hamburg 2001.

Noer, Afrin C./Notosusanto, Nugroho/Dwipayana, Gufran, Pengkhianatan G30S/PKI, DVD, 271 min., Indonesien 1984.

O. A., Hard-line groups to protest 1965 apology at State Palace, in: The Jakarta Post, 2.6.2016, [http://www.thejakartapost.com/news/2016/06/02/hard-line-groups-to-protest-1965-apology-at-state-palace.html], eingesehen 15.8.2017. 
O. A., "Wir haben 58000 politische Gefangene“, in: Der Spiegel, Nr. 27, 29.6.1970, S. 9798, hier S. 97, [http://www.spiegel.de/spiegel/print/d-44931081.html], eingesehen 11.8.1970.

Oppenheimer, Joshua/Sørensen, Signe Byrge, The Act of Killing, DVD, 159 min., Dänemark/Norwegen/Großbritannien 2012.

Oppenheimer, Joshua/Sørensen, Signe Byrge, The Look of Silence, DVD, 103 min., Dänemark 2014.

Parlina, Ina/Sundaryani, Fedina S., Jokowi rejects apology, promotes stability, in: The Jakarta Post, 2.10.2015, [http://www.thejakartapost.com/news/2015/10/02/jokowirejects-apology-promotes-stability.html], eingesehen 15.8.2017.

Peeters, Flor, Der Indonesische Kommunismus und der gescheiterte Staatsstreich, in: Zeitschrift für Politik 14 (1967), Heft 2, S. 187-202.

Poeze, Harry A., The Cold War in Indonesia, 1948, in: Journal of Southeast Asian Studies 40 (2009), Heft 3, S. 497-517.

Ricklefs, Merle C., A History of Modern Indonesia since c. 1200. Third Edition, Basingstoke 2001.

Roosa, John, Pretext for Mass Murder. The September 30th Movement and Suharto's Coup d'État in Indonesia, Madison 2006.

Schlereth, Einar/Bintang, Batjo Daeng, Indonesien. Analyse eines Massakers, Frankfurt a. M. 1970.

Sejarah, Lembaga, 40 hari kegagalan 'G-30-S' 1 Oktober-10 November 1965, Jakarta 1966.

Swift, Ann, The Road to Maidun. The Indonesian Communist Uprising of 1948, Singapur 2010.The Editors of Encyclopaedia Britannica, Maidun Affair, Encyclopaedia Britannica, 20.1.1998, [https://www.britannica.com/event/Madiun-Affair], eingesehen 20.3.2017.

Ufen, Andreas, Vergangenheitspolitik in Indonesien. Die Massaker von 1965-1966, in: GIGA Focus Asien (2014), Heft 3.

U.S. Bureau of the Census, World Population 1979. Recent Demographic Estimates for the Countries and Regions of the World, Washington D. C. 1980.

Vltchek, Andre, Indonesia. Archipelago of Fear, London 2012.

Waldschmidt, Anne/Klein, Anne u. a., Diskurs im Alltag - Alltag im Diskurs: Ein Beitrag zu einer empirisch begründeten Methodologie sozialwissenschaftlicher Diskursforschung, in: Forum Qualitative Sozialforschung/Forum: Qualitative Social Research 8 (2007), Heft 2, [http://nbn-resolving.de/urn:nbn:de:0114-fqs0702156], eingesehen 3.7.2017.

White, Hayden, Metahistory. Die historische Einbildungskraft im 19. Jahrhundert, Frankfurt a. M. 1991. 


\section{Zeitungsartikel Der Spiegel-Analyse 1, chronologisch}

O. A., Prächtiger Kerl, in: Der Spiegel, Nr. 42, 13.10.1965, S. 114-116, [http://www.spiegel. de/spiegel/print/d-46274703.html], eingesehen 11.8.2017.

O. A., Neues kom, in: Der Spiegel, Nr. 44, 27.10.1965, S. 132-133, [http://www.spiegel.de/ spiegel/print/d-46274703.html], eingesehen 11.8.2017.

O. A., Büffel in Flammen, in: Der Spiegel, Nr. 45, 3.11.1965, S. 139-141, [http://www.spiegel.de/spiegel/print/d-46274815.html], eingesehen 11.8.2017.

O. A., Macht der Messer, in: Der Spiegel, Nr. 1, 3.1.1966, S. 58, [http://www.spiegel.de/ spiegel/print/d-46265207.html], eingesehen 11.8.2017.

O. A., Neun Leben, in: Der Spiegel, Nr. 11, 7.3.1966, S. 88, [http://www.spiegel.de/spiegel/ print/d-46265938.html], eingesehen 11.8.2017.

O. A., Louis Barcata, „Ich selbst bin wie ein Kind”, in: Der Spiegel, Nr. 13, 21.3.1966, S. 146150, [http://www.spiegel.de/spiegel/print/d-46266100.html], eingesehen 11.8.2017.

${ }^{*}$ O. A., Jahr des Pferdes, in: Der Spiegel, Nr. 13, 21.3.1966, S. 143-144, [http://www.spiegel.de/spiegel/print/d-46266099.html], eingesehen 11.8.2017.142

${ }^{*}$ O. A., Macht der Geisha, in: Der Spiegel, Nr. 19, 2.5.1966, S. 136-138, [http://www.spiegel.de/spiegel/print/d-46407119.html], eingesehen 11.8.2017.

O. A., Frauen töten die Generäle, in: Der Spiegel, Nr. 20, 9.5.1966, S. 99-100, [http://www. spiegel.de/spiegel/print/d-46407207.html], eingesehen 11.8.2017.

*O. A., Traum vom Sprung, in: Der Spiegel, Nr. 36, 29.8.1966, S. 66, [http://www.spiegel. de/spiegel/print/d-46414101.html], eingesehen 11.8.2017.

O. A., Jungfrau in der Räuberhöhle, in: Der Spiegel, Nr. 39, 19.9.1966, S. 144-145, [http:// www.spiegel.de/spiegel/print/d-46414594.html], eingesehen 11.8.2017.

O. A., Gefährliche Farbe, in: Der Spiegel, Nr. 44, 24.10.1966, S. 132-133, [http://www.spiegel.de/spiegel/print/d46414849.html], eingesehen 11.8.2017.

O. A., Höhle der Krokodile, in: Der Spiegel, Nr. 45, 31.10.1966, S. 157-158, [http://www. spiegel.de/spiegel/print/d-46414952.html], eingesehen 11.8.2017.

*O. A., Brutal sein, in: Der Spiegel, Nr. 48, 21.11.1966, S. 114-131, [http://www.spiegel.de/ spiegel/print/d-46414952.html], eingesehen 11.8.2017.

O. A., Tarzie Vittachi, In Socken floh der Präsident zum Helikopter, in: Der Spiegel, Nr. 10, 27.2.1967, S. 109-120, [http://www.spiegel.de/spiegel/print/d-46415271.html], eingesehen 11.8.2017.

O. A., Nummer ab, in: Der Spiegel, Nr. 10, 27.2.1967, S. 106-107, [http://www.spiegel.de/ spiegel/print/d-46394457.html], eingesehen 11.8.2017.

142 Die mit * markierten Artikel thematisieren die indonesische Politik bzw. die Ereignisse von 1965/66 am Rande. 


\section{Zeitungsartikel Der Spiegel-Analyse 2, chronologisch}

*O. A., IUnsere Gedanken sind tot", in: Der Spiegel, 17, 1.7.1990, S. 130-133, [http://www. spiegel.de/spiegel/print/d-13487469.html], eingesehen 11.8.2017.

${ }^{*}$ O. A., Kapitel V - Stalins Erben. Verfall einer Weltmacht, in: Spiegel Special, Nr. 4, 1.12.1991, o. S., [http://www.spiegel.de/spiegel/spiegelspecial/d-49407551.html], eingesehen 11.8.2017.

*O. A., Rotes Gespenst, in: Der Spiegel, Nr. 35, 26.8.1996, S. 129, [http://www.spiegel.de/ spiegel/print/d-9086109.html], eingesehen 11.8.2017.

${ }^{*}$ O. A., Die Insel der Friedhöfe, in: Der Spiegel, Nr. 43, 21.10.1996, S. 179-187, [http:// www.spiegel.de/spiegel/print/d-9108473.html], eingesehen 11.8.2017.

*O. A., Christoph Pauly „,Wir brauchen mehr als Aspirin“, in: Der Spiegel, Nr. 4, 19.1.1998, S. 82-83, [http://www.spiegel.de/spiegel/print/d-7810108.html], eingesehen 11.8.2017.

*O. A., Der sterbende Elefant, in: Der Spiegel, Nr. 21, 18.5.1998, S. 166-169, [http://www. spiegel.de/spiegel/print/d-7892910.html], eingesehen 11.8.2017.

${ }^{*}$ O. A., Sturz in die Finsternis, in: Der Spiegel, Nr. 50, 7.12.1998, S. 200-202, [http://www. spiegel.de/spiegel/print/d-8440705.html], eingesehen 11.8.2017.

*O. A.,,Wie geht's dir, alte Tante Java?", in: Der Spiegel, Nr. 23, 7.6.1999, S. 186-187, [http:// www.spiegel.de/spiegel/print/d-13667566.html], eingesehen 11.8.2017.

${ }^{*}$ O. A., Abdul Haris Nasution, in: Der Spiegel, Nr. 39, 25.9.2000, S. 310, [http://www.spiegel.de/spiegel/print/d-17436657.html], eingesehen 11.8.2017.

${ }^{*}$ O. A., Suharto, in: Der Spiegel, Nr. 6, 2.2.2008, S. 146, [http://www.spiegel.de/spiegel/ print/d-55668252.html], eingesehen 11.8.2017.

O. A., Ayu Utami, Sturz der Henker, in: KulturSpiegel, Nr. 11, 28.10.2013, o. S., [http://www. spiegel.de/spiegel/kulturspiegel/d-117763264.html], eingesehen 11.8.2017.

O. A., Joshua Oppenheimer, in: Der Spiegel, Nr. 10, 1.3.2014, S. 136, [http://www.spiegel. de/spiegel/print/d-125300729.html], eingesehen 11.8.2017.

${ }^{*} O$. A., Der Triumph der Killer, Interview mit Klaus Theweleit, in: Der Spiegel, Nr. 20, 9.5.2015, S. 136-139, [http://www.spiegel.de/spiegel/print/d-134879030.html], eingesehen 11.8.2017.

*O. A., Das Reich des sanften Islam, in: Der Spiegel, Nr. 32, 1.8.2015, S. 80-83, [http:// www.spiegel.de/spiegel/print/d-137878522.html], eingesehen 11.8.2017. 
Andreas Fink, Studium der Politikwissenschaft in Wien sowie Geschichte und Deutsch auf Lehramt an der Leopold-Franzens-Universität Innsbruck.

\section{Zitation dieses Beitrages}

Andreas Fink, "Die Rache der Antikommunisten war nicht minder grausam." Die Ereignisse von 1965/66 in der indonesischen Erinnerungskultur und ihre Rezeption im deutschsprachigen Raum, in: historia.scribere 10 (2018), S. 79-109, [http://historia.scribere.at], eingesehen 19.6.2018 (=aktuelles Datum).

(C) Creative Commons Licences 3.0 Österreich unter Wahrung der Urheberrechte der Autorlnnen. 
